AperTO - Archivio Istituzionale Open Access dell'Università di Torino

\title{
A General Theory of Subjective Mixtures
}

\section{This is the author's manuscript}

Original Citation:

Availability:

This version is available http://hdl.handle.net/2318/1690404

since 2020-05-03T11:27:30Z

Published version:

DOI:10.1016/j.jet.2020.105056

Terms of use:

Open Access

Anyone can freely access the full text of works made available as "Open Access". Works made available under a Creative Commons license can be used according to the terms and conditions of said license. Use of all other works requires consent of the right holder (author or publisher) if not exempted from copyright protection by the applicable law. 


\section{Collegio Carlo Alberto}

\section{A general theory of subjective mixtures}

Paolo Ghirardato, Daniele Pennesi

No. 573

December 2018 (Revised, March 2020)

\section{Carlo Alberto Notebooks}

www.carloalberto.org/research/working-papers

(C) 2018 by Paolo Ghirardato, Daniele Pennesi. Any opinions expressed here are those of the authors and not those of the Collegio Carlo Alberto. 


\title{
A general theory of subjective mixtures*
}

\author{
Paolo Ghirardato $^{\S}$ Daniele Pennesi ${ }^{\llbracket}$ \\ June 2018 \\ This version: March 2020
}

\begin{abstract}
We provide, and characterize behaviorally, a framework for constructing subjective mixtures which requires neither the Certainty Independence nor the Monotonicity axiom, replacing them with much weaker "local" properties. As we also show by means of examples, this framework provides a purely subjective foundation to most of the recent preference models which employ the Anscombe-Aumann setting. It also allows a subjective formulation of a preference for ambiguity hedging, and as a consequence allows the distinction of the notions of ambiguity aversion and preference for randomization.
\end{abstract}

\section{Introduction}

L.J. Savage's axiomatization of the Subjective Expected Utility (SEU) model (Savage, 1954) has justly been hailed as a "crowning achievement" of decision theory. Its conciseness and beauty, however, came at a cost, and its extensions formulated to capture non-expected utility behavior have been very few (the closest being Gilboa's axiomatization of Choquet Expected Utility in Gilboa (1987)). On the other hand, the SEU axiomatization of Anscombe and Aumann (1963, henceforth AA), as reformulated by Fishburn (1970), has been extended in many different directions. The reason for this popularity is that the vector space structure induced by a randomizing device in the AA approach makes it much easier to formulate generalizations of axioms such as independence, which correspond directly to properties of the preference representation. However, a conceptual problem with the AA approach is that it builds a vector space structure via a randomizing device which is "objective," in the sense that all actors agree on its mixtures. For this

\footnotetext{
*An earlier version of this paper was titled "A new subjective spin on roulette wheels." We thank an Editor of this journal and three anonymous referees, as well as audiences at three conferences, for helpful and stimulating comments.

${ }^{\S}$ DESOMAS and Collegio Carlo Alberto, University of Torino, email: paolo.ghirardato@unito.it

'DESOMAS, University of Torino, email: daniele.pennesi@unito.it
} 
reason, Ghirardato, Maccheroni, Marinacci, and Siniscalchi (2001, 2003, henceforth GMMS) ${ }^{1}$ proposed a framework to define mixtures without the presence of such randomizing device. ${ }^{2}$ Within their framework, it is possible to construct a (subjective) vector space structure that can be used to formulate general AA-style axioms; this for instance allows the formulation of models which accommodate both the Ellsberg and Allais paradoxes (see Dean and Ortoleva, 2017).

However, the proposal of GMMS suffers from some limitations that over time have become increasingly apparent. Specifically, the GMMS construction assumed the traditional Monotonicity axiom of Gilboa and Schmeidler (1989), and it also depends on their Certainty Independence axiom. ${ }^{3}$ Monotonicity entails a separability property of preferences, which has been recently discussed theoretically and experimentally by Schneider and Schonger (2015). Moreover, it rules out several recent decision models, such as the Mean-Dispersion Preferences of Grant and Polak (2013), the dual utility model of Bommier (2017), the generalized convex representation of Cheridito, Delbaen, Drapeau, and Kupper (2015), and the correlation misperception model of Ellis and Piccione (2017). As to Certainty Independence, it is by now well understood that it imposes strong restrictions on the decision maker's attitudes as they change over wealth levels; see Baillon and Placido (2019) and the discussion in Cerreia-Vioglio, Ghirardato, Maccheroni, Marinacci, and Siniscalchi (2011a, henceforth CGMMS). This rules out models such as Variational Preferences (Maccheroni, Marinacci, and Rustichini, 2006) and the "Smooth Ambiguity" model (Klibanoff, Marinacci, and Mukerji, 2005).

In this paper we provide a framework for constructing subjective mixtures which requires neither Monotonicity nor Certainty Independence, replacing these axioms with much weaker "local" properties. This framework provides a purely subjective foundation to all the mentioned models, as well as those discussed in GMMS. As we illustrate by means of several examples, the basic preference assumptions needed to apply our construction add very little structure to that required by the model that one wants to characterize. ${ }^{4}$

\footnotetext{
${ }^{1}$ When we need to distinguish between the two papers, we will use GMMSa to refer to Ghirardato et al. (2001) and GMMSb to refer to Ghirardato et al. (2003).

${ }^{2}$ There are, of course, many papers that characterize non-expected utility preferences in purely subjective environments, without explicitly constructing a mixture space structure. For instance, Casadesus-Masanell, Klibanoff, and Ozdenoren (2000) and Alon and Schmeidler (2014) for the Maxmin EU model and Nakamura (1990) for the Choquet EU model.

${ }^{3}$ Monotonicity requires that if $f(s) \succcurlyeq g(s)$ for every state $s$, then $f \succcurlyeq g$. Certainty Independence requires that for all acts $f$ and $g$, all outcomes $x \in X$, and all $\lambda \in[0,1], f \succcurlyeq g$ implies $\lambda f+(1-\lambda) x \succcurlyeq \lambda g+(1-\lambda) x$.

${ }^{4}$ In Ghirardato and Pennesi (2019) we show that in those situations in which the space of outcomes has a productspace structure, subjective mixtures can be derived by an alternative method which makes no assumptions on the existing uncertainty.
} 
The starting point for our analysis is GMMS' method for identifying a "preference average" of two outcomes $x$ and $y$. Given an event $E$ and a bet $v E w$-which pays outcome $v$ if event $E$ obtains and outcome $w$ otherwise- let $c_{\nu E w}$ denote its certainty equivalent. For an event $E$ which is neither certain nor impossible, we call the outcome $z$ a E-preference average of $x$ and $y$, and denote $z=\frac{1}{2} x \oplus \frac{1}{2} y$, if $z$ satisfies the following indifference:

$$
x E y \sim c_{x E z} E c_{z E y}
$$

That is, since $x E y \sim c_{x E x} E c_{y E y}$, we can replace $z$ for $x$ and $y$ in the bets on the right-hand side of the indifference without affecting the DM's evaluation of the bet $x E y$. To provide an intuition for this definition, based on an idea of von Neumann (1951), suppose that the event $E$ is a result in a twice repeatable experiment. If the repetitions are performed independently, then regardless of our beliefs about $E$, the sequences $\left(E, E^{c}\right)$ and $\left(E^{c}, E\right)$ are equally likely ${ }^{5}$. Thus, a bet which conditionally on observing either $\left(E, E^{c}\right)$ or $\left(E^{c}, E\right)$ — pays $x$ if $\left(E, E^{c}\right)$ and $y$ if $\left(E^{c}, E\right)$ is like a 1/2:1/2 bet with payoffs $x$ and $y$; i.e., a bet on a "fair coin." So a decision maker (DM) who is indifferent between such bet and a bet which pays $z$ conditionally on the same two sequences deems the outcome $z$ a 1/2:1/2 average of $x$ and $y$. Expression (1) adapts this construction to the case in which $E$ is an event in the decision problem's state space, thus non-repeatable, using certainty equivalents of bets on $E$ as a tool to reproduce the effects of the repetition of the experiment on $E$.

Suppose now that the DM's preferences are represented by a cardinal functional $V$ which, when evaluating bets on the event $E$-i.e., acts which pay outcome $x$ if event $E$ obtains and outcome $y$ otherwise (with $x \succcurlyeq y$ )— takes the following form:

$$
V(x E y)=\rho V(x)+(1-\rho) V(y)
$$

for some $\rho \in(0,1)$. Then for such bets (1) implies that $V(z)=V\left(\frac{1}{2} x \oplus \frac{1}{2} y\right)=\frac{1}{2} V(x)+\frac{1}{2} V(y)$. So, $E$ preference averages are also utility midpoints according to the representation $V$. In view of this, the mixture operator $\oplus$ over outcomes defined via (1) has the same properties as the "objective" mixture of Anscombe and Aumann, and the vector space structure it induces can be analogously applied to leverage functional-analytic tools. However, our structure is completely subjective.

\footnotetext{
${ }^{5}$ Thus, it would be enough for the experiment sequence to be exchangeable, rather than i.i.d.
} 
We remark that the representation in Eq. (2), for which we provide a behavioral characterization, only holds for bets "on" E, but not for bets on other events, or for non-binary acts. This is why we call $V$ a "locally biseparable" representation. We do not assume any further structure for $V$; in particular, unlike GMMS, we do not require that $V$ be monotonic or that it satisfies further separability properties, such as Certainty Independence. The additional scope of our analysis is illustrated by several examples of preferences which are locally biseparable but do not satisfy the assumptions of GMMS. ${ }^{6}$

We conclude the paper with two applications of subjective mixtures to different issues in decision theory. First, we show how to introduce a notion of "ex-ante" mixture in our setting. This allows us to model DMs who may not be indifferent towards the subjective timing of randomization, and address Raiffa (1961)'s critique of ambiguity aversion. Second, we discuss how comparing subjective mixtures to those obtained via an objective randomization device allows us to disentangle two potentially distinct behavioral traits that since the seminal work of Schmeidler (1989) have been identified: ambiguity hedging and preference for randomization. This identification also plays a major role in the recent debate on the effectiveness of the Random Lottery Incentive Scheme in experiments on ambiguity (see, e.g., Bade, 2015). There are however doubts as to the validity of the identification, both from a theoretical perspective (see, e.g., Eichberger and Kelsey, 1996) and from an experimental perspective (see, e.g., Dominiak and Schnedler, 2011). By severing the ties between ambiguity aversion and preference for randomization, our framework provides the necessary flexibility to discuss all these questions.

The structure of the paper is as follows. After some preliminary material in Section 2, in Section 3 we introduce and characterize behaviorally locally biseparable preferences, showing that Expression (1) identifies utility midpoints. Section 4 contains examples of preferences which have a locally biseparable representation. Sections 5.1 and 5.2 contain the discussions on ex-ante mixtures and on objective randomization. The Appendices contain some additional material and all the proofs of the results in the paper. The Supplementary Appendices contain more additional material, in particular the consequences of further restrictions on the global properties of preferences, as well as an application to models with menus of outcomes such as Dekel, Lipman, and Rustichini (2009) and Gul and Pesendorfer (2001).

\footnotetext{
${ }^{6}$ In general, the construction of $\oplus$ can depend on the event $E$. But even if we additionally require that $\oplus$ be invariant across events for which Eq. (2) holds, our approach imposes looser constraints than GMMS (see Appendix B).
} 


\section{Preliminaries}

We consider an arbitrary state space $S$, an algebra $\Sigma$ of subsets of $S$ which represents events, and a set of outcomes $X$. $\mathscr{F}$ denotes the set of all simple acts; i.e., finite-valued $\Sigma$-measurable functions $f: S \rightarrow X$. As customary, we notationally identify an outcome $x \in X$ with the constant act $f$ such that $f(s)=x$ for all $s \in S$. Throughout the paper, $X$ is assumed to satisfy the following:

Structural assumption: $X$ is a connected and separable topological space with topology $\tau$. $^{7}$

Given a functional $V: \mathscr{F} \rightarrow \mathbb{R}$, we say that: $V$ represents a binary relation $\succcurlyeq$ on $\mathscr{F}$ if $f \succcurlyeq g$ iff $V(f) \geq V(g)$, that $V$ is nontrivial if there are $f, g \in \mathscr{F}$ such that $V(f)>V(g)$, that $V$ is constantbounded if for any $f \in \mathscr{F}, V(x) \geq V(f) \geq V(y)$ for some $x, y \in X$, and that $V$ is subcontinuous if $\lim _{\alpha} V\left(f_{\alpha}\right)=V(f)$ whenever $\left\{f_{\alpha}\right\}_{\alpha \in D} \subseteq \mathscr{F}$ is a net that pointwise converges to $f \in \mathscr{F}$, and such that all $f_{\alpha}$ 's and $f$ are measurable with respect to the same finite partition. (Notice that this implies that $\left.u \equiv V\right|_{X}$ is $\tau$-continuous.) Given representations $V$ and $V^{\prime}$, we write $V \approx V^{\prime}$, if there are $a, b \in \mathbb{R}$ such that $a>0$ and $V^{\prime}=a V+b$. In this case, we say that $V$ and $V^{\prime}$ are cardinally equivalent.

Finally, we use $x \succcurlyeq\left\{z, z^{\prime}\right\}$ (resp. $\left\{z, z^{\prime}\right\} \succcurlyeq y$ ) as a short-hand for $x \succcurlyeq z$ and $x \succcurlyeq z^{\prime}$ (respectively $z \succcurlyeq y$ and $\left.z^{\prime} \succcurlyeq y\right)$

\section{A foundation for subjective mixtures}

\subsection{Utility midpoints and locally biseparable preferences}

As previewed in the Introduction, our approach to the identification of utility midpoints is based on the assumption that the preferences over bets on a specific event have a suitably separable representation. In this section, we first define midpoints and then introduce the required preference model, while the behavioral foundation for such model is deferred to the following section.

For now, we assume that the preference relation $\succcurlyeq$ is a non-trivial weak order (complete and transitive) and that any $f \in \mathscr{F}$ has a certain equivalent; i.e., a $c_{f} \in X$ such that $f \sim c_{f}$. Given such $\succcurlyeq$, an event $A \in \Sigma$ is said to be null (resp. universal) if $y \sim x A y$ (resp. $x \sim x A y$ ) for every $x>y$. We denote by $\Sigma^{\prime}$ the family of neither null nor universal events $E \in \Sigma$.

We can now recall the notion of preference average discussed in the Introduction:

\footnotetext{
${ }^{7}$ As it is well-known, $\tau$ induces the product topology on the set $X^{S}$ of all functions from $S$ into $X$. In this topology, a net $\left\{f_{\alpha}\right\}_{\alpha \in D} \subseteq X^{S}$ converges to $f \in X^{S}$ if and only if $f_{\alpha}(s) \stackrel{\tau}{\longrightarrow} f(s)$ for all $s \in S$ (remember that $S$ is arbitrary).
} 
Definition 1. Given $x \succcurlyeq y$ and an event $E \in \Sigma^{\prime}$, we call $z \in X$ an $E$-preference average of $x$ and $y$ if $z$ satisfies $x \succcurlyeq z \succcurlyeq y$ and

$$
x E y \sim c_{x E z} E c_{z E y}
$$

While Definition 1 is applicable to any preference $\succcurlyeq$ which satisfies the conditions above, our results apply to the subset of such preferences which satisfy the following definition:

Definition 2. Given $E \in \Sigma$, a binary relation $\succcurlyeq$ on $\mathscr{F}$ is said to be E-biseparable if $\succcurlyeq$ has a representation $V$ that is nontrivial, constant-bounded and subcontinuous and there exists $a \rho_{E} \in(0,1)$ such that for any $x, y \in X$ satisfying $x \succcurlyeq y$, if we let $u=V_{\mid X}$,

$$
V(x E y)=\rho_{E} u(x)+\left(1-\rho_{E}\right) u(y)
$$

The binary relation $\succcurlyeq$ is said to be locally biseparable if it is E-biseparable for some $E \in \Sigma$.

The features of a locally biseparable preference which follow from Eq. (3) and are important for our purposes are the following: 1) Since the DM's willingness to bet on $E$ satisfies $\rho_{E} \in(0,1)$, the event $E$ satisfies $x>x E y>y$ for all $x>y ;^{8}$ in particular, $E \in \Sigma^{\prime}$, and it can be used to define preference averages. 2) For the same reason, the representation $V$ is cardinal; thus, $u$ is also cardinal and the $1 / 2: 1 / 2$ mixtures it produces are well-defined. 3) In contrast, $\rho_{E}$ is invariant to the positive affine transformations of $V$, and hence unique.

GMMSb differs from our approach in assuming that $\succcurlyeq$ has a fully biseparable representation. That is, Eq. (3) holds for all events and not just for the event $E$. In contrast, GMMSa also characterize (in their Lemma 1) a "local" form of biseparability. However, they assume that $V$ is monotonic and also that $\succcurlyeq$ is $E^{c}$-biseparable (i.e. Eq. (3) holds for bets on $E^{c}$ with a weight $\rho_{E^{c}} \in(0,1)$ ).

The following straightforward result explains our usage of "preference average" in Definition 1.

Proposition 1. Suppose that $\succcurlyeq$ is E-biseparable then, for any $x, y \in X$ satisfying $x \succcurlyeq y$, there is an E-preference average of $x$ and $y$. The payoff $z \in X$ is an E-preference average of $x$ and $y$ if and only if $z$ is a midpoint according to $u$. That is,

$$
u(z)=\frac{1}{2} u(x)+\frac{1}{2} u(y)
$$

\footnotetext{
${ }^{8}$ That is, $E$ is an "essential" event in the sense of Ghirardato and Marinacci (2001).
} 
In light of Proposition 1, we henceforth refer to a $z$ satisfying the conditions of Definition 1 as the utility midpoint of $x$ and $y$, and denote

$$
z \equiv \frac{1}{2} x \oplus \frac{1}{2} y
$$

Given such notion, we can then iterate it to find, say, $\frac{1}{2} x \oplus \frac{1}{2}\left[\frac{1}{2} x \oplus \frac{1}{2} y\right]$, which corresponds to a $\frac{3}{4}: \frac{1}{4}$ utility average of $x$ and $y$, and which we denote by $\frac{3}{4} x \oplus \frac{1}{4} y$. Exploiting the continuity of $V$ over $X$, we can then apply standard arguments (see Appendix C in Ghirardato, Maccheroni, Marinacci, and Siniscalchi, 2002) to identify $\lambda x \oplus(1-\lambda) y$ for any $\lambda \in[0,1]$ and all $x, y \in X$. The subjective mixture of acts $f$ and $g$ is now defined in the usual, state-by-state, fashion:

Definition 3. Given $f, g \in \mathscr{F}$ and $\lambda \in[0,1]$, the subjective mixture $\lambda f \oplus(1-\lambda) g$ is an act $h \in \mathscr{F}$ such that $h(s)=\lambda f(s) \oplus(1-\lambda) g(s)$ for every $s \in S$.

It follows from the definition of $\oplus$ that for every $f, g \in \mathscr{F}$ and $s \in S$ and for all $\lambda \in[0,1]$,

$$
u[(\lambda f \oplus(1-\lambda) g)(s)]=\lambda u(f(s))+(1-\lambda) u(g(s))
$$

Hence, subjective mixtures behave as the AA "objective" mixtures in the traditional AA model: they allow us to obtain the convex combination of any two utility profiles. Even in the presence of "objective" mixtures, the vector space structure induced by subjective mixtures is robust to violations of expected utility (see the discussion in Section 5.2).

The identification of subjective mixtures is thus achieved without imposing global separation properties such as Certainty Independence or Monotonicity. This allows to build subjective mixtures for a large class of preference models; see the examples in Section 4 and Remark 1.

The flip side of this generality is that the mixture structure thus identified may depend on the event $E$ with respect to which $\succcurlyeq$ has a biseparable representation. Given two events $E, F \in \Sigma^{\prime}$, suppose that the preference $\succcurlyeq$ is at the same time $E$ - and $F$-biseparable. It is possible that the respective representations $V_{E}$ and $V_{F}$, when restricted to the constant acts in $X$, are ordinally but not cardinally equivalent. That is, some utility midpoints according to $V_{E}$ may not be indifferent to those induced by $V_{F}$. Intuitively, this might be ruled out by requiring that $E$ and $F$ induce, modulo indifference, the same preference averages: ${ }^{9}$

\footnotetext{
${ }^{9}$ We observe that the following definition (as Definition 1 ) is well-posed for any $\succcurlyeq$ that is a non-trivial weak order
} 
Definition 4. Given $E, F \in \Sigma^{\prime}$ and a binary relation $\succcurlyeq$, we say that $E$ and $F$ are average-equivalent if, for all $x \succcurlyeq\left\{z, z^{\prime}\right\} \succcurlyeq y$,

$$
x E y \sim c_{x E z} E c_{z E y} \text { and } x F y \sim c_{x F z^{\prime}} F c_{z^{\prime} F y} \Rightarrow z \sim z^{\prime}
$$

The following result shows that the intuition is correct: If $E$ and $F$ are average-equivalent, then the utility midpoints according to $V_{E}$ and $V_{F}$ are the same, and thus $V_{E}$ and $V_{F}$ (and hence $u_{E}$ and $u_{F}$ ) are cardinally equivalent.

Lemma 1. Given $E, F \in \Sigma^{\prime}$, suppose that $\succcurlyeq i s ~ E$ - and $F$-biseparable. Then $E$ and $F$ are averageequivalent if and only if $V_{E} \approx V_{F}$.

So average-equivalent events are indifferent for the purposes of our method. Indeed, if all events with respect to which $\succcurlyeq$ has a biseparable representation are average-equivalent (see Axiom CB in Appendix B), then our method will yield the same mixtures independently of the event chosen to identify them. This condition is weaker than those adopted by GMMS, ${ }^{10}$ which imply that all events $F \in \Sigma^{\prime}$ are average-equivalent and induce $F$-biseparable representations. For this reason, GMMS do not present a result analogous to Lemma 1.

\subsection{Behavioral characterization}

In this section, we show which preference conditions correspond to local biseparability. We start with an axiom which bundles some standard requirements on preferences, including nontriviality and boundedness. $^{11}$

Axiom (Preference Order - P). $\succcurlyeq$ is a complete and transitive relation on $\mathscr{F}$ which also satisfies: 1 ) there are $f, g \in \mathscr{F}$ such that $f>g$, 2) for each $f \in \mathscr{F}$, there exist $x, y \in X$ such that $x \succcurlyeq f \succcurlyeq y$.

The second axiom is a continuity property, taken from Ghirardato and Marinacci (2001). Notice that all acts involved are measurable with respect to the same finite partition of $S$, making the condition rather weak.

\footnotetext{
with certainty equivalents.

${ }^{10} \mathrm{GMMSa}$ assumes local biseparability plus a variant of Certainty Independence (replacing $\succcurlyeq$ with $\sim$ ) with respect to subjective mixtures. GMMSb assumes full biseparability, as discussed above.

${ }^{11}$ Boundedness is usually omitted as (for simple acts) it is implied by Monotonicity.
} 
Axiom (Continuity - C). Let $\left\{f_{\alpha}\right\}_{\alpha \in D}$ be a net in $\mathscr{F}$ that pointwise converges to $f \in \mathscr{F}$ and such that all $f_{\alpha}$ and $f$ are measurable w.r.t. the same finite partition of $(S, \Sigma)$. Given $g \in \mathscr{F}$, if $f_{\alpha} \succcurlyeq g$ (resp. $g \succcurlyeq f_{\alpha}$ ) for all $\alpha \in D$, then $f \succcurlyeq g($ resp. $g \succcurlyeq f)$.

Alongside the Structural Assumption, these two axioms imply the existence of certainty equivalents (see Lemma 3 in the Appendix). Indeed, standard arguments then imply the existence of a representation $V$ of $\succcurlyeq$ which is non-constant, constant-bounded and sub-continuous. However, such $V$ is only unique up to monotonic increasing transformations, and cardinality is necessary in order to meaningfully define a utility midpoint of two outcomes.

Moreover, we now show by example that the identification of utility midpoints via $E$-preference averages is only possible if the DM's willingness to bet on $E$ is independent of the bet's stakes.

Example 1. Let $S=\left\{s_{1}, s_{2}\right\}, X=[0,10]$. Suppose that $\succcurlyeq$ is represented by a $V$ which satisfies $V(x)=$ $u(x)=x$, and for $E=\left\{s_{1}\right\}$ it satisfies, with $\rho_{x}(E)=0.2+0.02(10-x)$ for $x \in X$,

$$
V(x E y)=1_{x \leq 5}[0.3 x+0.7 y]+1_{x>5}\left[\rho_{x}(E) x+\left(1-\rho_{x}(E)\right) y\right]
$$

Clearly, the willingness to bet on $E$ is not stake-independent, so that the preference is not $E$ biseparable. And, indeed, E-preference averages are not utility midpoints. For example, if we consider $x=6$ and $y=2$, whose utility midpoint is clearly 4, we can show (see Supplementary Appendix ?? for all details on this example)

$$
6 E 2 \sim c_{6 E 3.84} E c_{3.84 E 2}
$$

That is, the $E$-preference average of $x$ and $y$ is 3.84. Therefore, for $x=6, y=2$ the "weight" assigned to $x$ by the $E$-preference average is slightly smaller than 0.5 . On the other hand, it can also be seen that if we take $x=4, y=2$ the $E$-preference average yields $z^{\prime}=3$ and the "weight" assigned to $x$ equals 0.5 (it can be seen in Eq. (5) that when both payoffs are lower than 5, the willingness to bet on $E$ is fixed at 0.3 and thus independent of the payoffs).

Our objective is therefore to identify properties on preferences which guarantee the stakeindependence of the willingness to bet and at the same time imply a cardinal representation. One obvious component of independence is given by the following property: 
Definition 5. An event $E \in \sum$ is betwise-monotonic, for all $x, y, z \in X: x \succcurlyeq y$ ifand only if $x E z \succcurlyeq y E z$ for any $z \preccurlyeq\{x, y\} ; x \succcurlyeq y$ if and only if $z E x \succcurlyeq z E y$ for any $z \succcurlyeq\{x, y\}$.

As its name suggests, this is a form of restricted (local) monotonicity, as it stipulates that the DM's preferences respect monotonicity only over the set of the bets "on" the event $E$ (i.e., acts $x E y$ such that $x \succcurlyeq y) .{ }^{12}$ It is also an independence/separation property, as it implies that when comparing bets on $E$, the evaluation of a payoff on one side of the bet does not depend on the alternative payoff on the other side (as long as we are comparing only bets on $E$ ).

It can be seen that, under nontriviality of $\succcurlyeq$, if $E$ is betwise-monotonic, then $E \in \Sigma^{\prime}{ }^{13}$ Moreover, in the presence of Axioms $\mathrm{P}$ and C, $E$-preference averages exist (for every $x \succcurlyeq y$ ) for every betwise-monotonic $E$. However, the existence of a betwise-monotonic event, while clearly necessary, is still not sufficient to obtain $E$-biseparability and to identify utility midpoints via $E$ preference averages. Refer again to Example 1: It can be seen (again, see Supplementary Appendix ??) that the event $E$ does satisfy betwise monotonicity.

We thus introduce an additional separation property.

Definition 6. An event $E \in \Sigma$ is ordered-bisymmetric if, for all $x, y, z, z^{\prime} \in X$ such that $x \succcurlyeq\left\{z, z^{\prime}\right\} \succcurlyeq$ $y \in X$, we have:

$$
c_{x E z} E c_{z^{\prime} E y} \sim c_{x E z^{\prime}} E c_{z E y}
$$

It is immediate to see that ordered bisymmetry of $E$ is necessary for $\succcurlyeq$ to have an $E$-biseparable representation. It is also easy to verify (see Supp. App. ??) that $E$ in Example 1 is not orderedbisymmetric (indeed, the preference represented by $V$ has no ordered-bisymmetric events).

To understand the behavioral content of this property, recall the discussion of preference averages in the Introduction. Suppose that the DM thinks of any "two-stage" bet like $c_{x E z} E c_{z^{\prime} E y}$ as follows: the "real experiment" $\left\{E, E^{c}\right\}$ is performed first; its payoffs are bets ( $x E z$ and $\left.z^{\prime} E y\right)$ whose payoffs depend on the outcome of a ficticious repetition of the experiment $\left\{E, E^{c}\right\}$. Requiring ordered bisymmetry of $E$ amounts to assuming that the DM considers the sequence (real experiment, ficticious experiment) exchangeable: Since the two "two-stage" bets $c_{x E z} E c_{z^{\prime} E y}$ and

\footnotetext{
${ }^{12}$ It should be pointed out that there is an aspect in which this property is slightly stronger than the standard Monotonicity axiom; the latter would only imply that if $x \succcurlyeq y$, then $x E z \succcurlyeq y E z$ and $z E x \succcurlyeq z E y$.

${ }^{13}$ As we show in Fact ?? in Supplementary Appendix ??, the condition that $E$ be neither null nor universal is necessary for the cardinal identification of $V$.
} 
$c_{x E z^{\prime}} E c_{z E y}$ only differ as to the payoff conditional on the pairs $\left(E, E^{c}\right)$ and $\left(E^{c}, E\right),{ }^{14}$ indifference reveals that she deems the pairs equally likely. So, when $E$ is ordered-bisymmetric, $E$-preference averages are isomorphic to the 1/2:1/2 mixtures in von Neumann (1951)'s "fair coin" construction.

Summing up, we have introduced and discussed two conditions on (preferences over bets on) $E$ which are necessary for $E$-biseparability. The following definition unifies them:

Definition 7 (Ordered Niceness - ON). An event $E \in \Sigma$ is ordered-nice if:

(a) E is betwise-monotonic

(b) E is ordered-bisymmetric

Our basic representation result, leveraging on results of Nakamura (1990), shows that (alongside Axioms $\mathrm{P}$ and $\mathrm{C}$ ) condition $\mathrm{ON}$ is also sufficient.

Proposition 2. Given $E \in \Sigma$, the binary relation $\succcurlyeq$ has an E-biseparable representation if and only if $\succcurlyeq$ satisfies Axioms $P$ and $C$, and the event $E$ satisfies $O N$.

The characterization of local biseparability follows immediately:

Corollary 1. The binary relation $\succcurlyeq$ is locally biseparable if and only if $\succcurlyeq$ satisfies Axioms P and C, and there exists an event $E \in \Sigma$ satisfying $O N$.

This result is more general than Lemma 1 of GMMSa. We do not assume Monotonicity and require only the Ordered Niceness of one event $E$ (and not of its complement $E^{c}$ ); an assumption which —-besides being necessary to the proper interpretation of $(E$-)preference averages— is quite weak. Our Axiom $\mathrm{C}$ is slightly stronger than theirs, but the two axioms are equivalent under the other assumptions in their result (see Lemma 31 in Ghirardato and Marinacci (2001)). The next section substantiates these claims by providing several examples of preferences which do not satisfy GMMS' conditions, but they are locally biseparable. ${ }^{15}$

On the other hand, one could be interested in understanding the consequences of imposing additional global restrictions on preferences. This exercise is performed in Supplementary Appendix ??; the following two remarks only briefly review two interesting cases.

\footnotetext{
${ }^{14}$ For both "two-stage" bets $x$ (resp. $y$ ) obtains if the sequence $(E, E)$ (resp. $\left(E^{c}, E^{c}\right)$ ) is observed. As to $z$ and $z^{\prime}$ : In $c_{x E z} E c_{z^{\prime} E y}, z$ obtains if $\left(E, E^{c}\right)$ while $z^{\prime}$ obtains if $\left(E^{c}, E\right)$. In $c_{x E z^{\prime}} E c_{z E y}, z$ obtains if $\left(E^{c}, E\right)$ while $z^{\prime}$ obtains if $\left(E, E^{c}\right)$.

${ }^{15}$ In contrast, we show in Appendix A that the second-order subjective expected utility (SOSEU) preferences of Grant, Polak, and Strzalecki (2009) have an ON event if and only if they are SEU.
} 
Remark 1. When we add Monotonicity to the axioms of Corollary 1, we obtain a fully subjective characterization of the Monotone, Bernoullian and Archimedean (MBA) model of CGMMS. MBA includes as special cases most of the recent models of ambiguity sensitive preferences, such as the Smooth Ambiguity model (Klibanoff et al., 2005), Variational Preferences (Maccheroni et al., 2006), Vector EU (Siniscalchi, 2009), and the Confidence Preferences model (Chateauneuf and Faro, 2009).

In MBA, subjective mixtures can be used to identify à la Bewley (2002) a set of beliefs $C \subseteq$ $\Delta(S)$ which may be interpreted as perceived ambiguity (Ghirardato, Maccheroni, and Marinacci (2004). ${ }^{16}$ CGMMS propose a definition of unambiguous event and show (Prop. 14) that an event $E$ is unambiguous if $p(E)=q(E)$ for all $p, q \in C$; that is, if all the perceived priors agree on the likelihood of $E$. It follows from their result and our Proposition 2 that MBA preferences are $E$ biseparable for all nontrivial (not null or universal) unambiguous events $E$; that is, all nontrivial unambiguous $E$ satisfy $\mathrm{ON}$.

Remark 2. Subjective mixtures allow us to formulate the following axiom, which adapts Schmeidler (1989)'s Uncertainty Aversion axiom to our setting:

Axiom (Ambiguity Hedging - AH). For all $f, g \in \mathscr{F}, f \sim g \Longrightarrow \frac{1}{2} f \oplus \frac{1}{2} g \succcurlyeq g$.

As our terminology suggests, this axiom has a natural interpretation: if $f$ and $g$ are indifferent, an act whose utility profile is the arithmetic average of the utility profiles of $f$ and $g$ provides a hedge to the ambiguity connected to each act and it will be weakly preferred to either act. On the other hand, Schmeidler's original formulation is interpreted more correctly as a preference for (objective) randomization, see the discussion in Section 5.2.

When we add Axiom AH to the axioms of Corollary 1 , the functional $V$ is also quasiconcave. ${ }^{17}$ Thus, Axiom AH and those characterizing MBA preferences (see Remark 1 above) provide a fully subjective characterization of the Uncertainty Averse Preferences of Cerreia-Vioglio, Maccheroni, Marinacci, and Montrucchio (2011b).

\footnotetext{
${ }^{16}$ For some applications see, e.g., Danan, Gajdos, Hill, and Tallon (2016) and Klibanoff, Mukerji, and Seo (2014).

${ }^{17}$ In the sense that, for all $f, g, h \in \mathscr{F}$, if $V(f) \geq V(h)$ and $V(g) \geq V(h)$, then $V(\alpha f \oplus(1-\alpha) g) \geq V(h)$ for all $\alpha \in[0,1]$.
} 


\section{Some examples}

In this section, we present some examples which help assessing the scope of our foundation of subjective mixtures. They also shed some light on the empirical content of the behavioral characterization of Section 3.2, in particular the existence of events satisfying ON. The examples also provide a comparison with the earlier work of GMMS, as they involve preferences which are locally biseparable and violate (global) Monotonicity or Certainty Independence. Appendix A shows an example of a well-known model which is not locally biseparable.

Example 2. Given a finite $S$, let $\succcurlyeq$ be represented by a mean-standard deviation functional $V$ with respect to some prior $p \in \Delta(S)$, a Bernoulli utility $u$, and $\tau \geq 0$ (i.e., a particular case of the dispersion aversion model of Grant and Polak (2013)):

$$
V(f)=\mathbb{E}_{p}[u \circ f]-\tau \sqrt{\sum_{s} p(s)\left(u(f(s))-\mathbb{E}_{p}[u \circ f]\right)^{2}}
$$

Assume that for some event $E$ in $\Sigma, p(E)=\frac{1}{2}$, then for all $x \succcurlyeq y,{ }^{18}$

$$
V(x E y)=\frac{1}{2}(1-\tau) u(x)+\frac{1}{2}(1+\tau) u(y)
$$

Thus, if $0 \leq \tau<1$, the preference $\succcurlyeq$ is locally biseparable with $\rho_{E}=\frac{1}{2}(1-\tau)$. More importantly, $\succcurlyeq$ satisfies Monotonicity only if $p(s)>\frac{\tau^{2}}{1+\tau^{2}}$ for all $s \in S$ (see Grant and Kajii, 2007). For example, with $|S|=10, p(s)=\frac{1}{10}$ for all $s \in S$, and $\tau=\frac{1}{2}$, the preference is locally biseparable but it is not monotone, as $p(s)<\frac{\tau^{2}}{1+\tau^{2}}$ for all $s \in S .^{19}$

Example 3. Consider a preference $\succcurlyeq$ represented by a non-monotonic Choquet integral as in Gilboa (1989) and De Waegenaere and Wakker (2001, Eq. 4). Assuming $|S|=n$ and denoting by

\footnotetext{
${ }^{18}$ This is shown as follows:

$$
\begin{aligned}
V(x E y) & =\frac{1}{2} u(x)+\frac{1}{2} u(y)-\tau \sqrt{\frac{1}{2}\left(u(x)-\frac{1}{2} u(x)-\frac{1}{2} u(y)\right)^{2}+\frac{1}{2}\left(u(y)-\frac{1}{2} u(x)-\frac{1}{2} u(y)\right)^{2}} \\
& =\frac{1}{2} u(x)+\frac{1}{2} u(y)-\tau \sqrt{\left(\frac{1}{2} u(x)-\frac{1}{2} u(y)\right)^{2}}=\frac{1}{2}(1-\tau) u(x)+\frac{1}{2}(1+\tau) u(y)
\end{aligned}
$$

${ }^{19} \succcurlyeq$ is also $E^{c}$-biseparable, but unless $\tau=0$ the corresponding $\rho_{E^{c}}$ is such that $\rho_{E}+\rho_{E^{c}} \neq 1$.
} 
$f_{i}$ the payoff of act $f$ in state $s_{i}$, the preferences $\succcurlyeq$ is represented by

$$
V(f)=p\left(s_{1}\right) u\left(f_{1}\right)+\sum_{i=2}^{n}\left[p\left(s_{i}\right) u\left(f_{i}\right)-\tau_{i}\left(u\left(f_{i-1}\right)-u\left(f_{i}\right)\right)^{+}\right]
$$

for some $p \in \Delta(S)$, with $(a)^{+}=\max (a, 0)$ and $\tau_{i} \in \mathbb{R}$. The function $V$ could be interpreted as representing the value of a stream of consumption when the DM has a weak form of reference dependence. If the payoff of period $i$ is smaller than the payoff of period $i-1$, there is a utility penalty (resp. premium) if $\tau_{i}>0$ (resp. $\tau_{i}<0$ ). Hence, the value of a sequence of payoffs is sensitive to intertemporal variations in consumption.

As shown in De Waegenaere and Wakker (2001), the representation $V$ can be written as a nonmonotonic Choquet integral; i.e., a Choquet integral with respect to a signed capacity. ${ }^{20}$ Given an event $E=\left\{s_{k}, \ldots, s_{n}\right\}$ with $k \in\{1, \ldots, n\}$, $\succcurlyeq$ is $E$-biseparable. Given $x \succcurlyeq y$, we have $V(x E y)=$ $p\left(E^{c}\right) u(y)+p\left(s_{k}\right) u(x)-\tau_{k}(u(y)-u(x))^{+}+\sum_{j=k+1} p\left(s_{j}\right) u(x)$, which implies

$$
V(x E y)=p(E) u(x)+(1-p(E)) u(y)
$$

Being represented by a Choquet integral, $\succcurlyeq$ satisfies Certainty Independence. However, it does not satisfy (global) Monotonicity (see De Waegenaere and Wakker, 2001, p. 52).

Example 4. Consider a preference $\succcurlyeq$ represented by the Smooth Ambiguity model of Klibanoff et al. (2005) and Seo (2009):

$$
V(f)=\phi^{-1}\left(\int_{\Delta(S)} \phi\left(\mathbb{E}_{p}[u \circ f]\right) d \pi(p)\right)
$$

where $\pi$ is a probability measure over $\Delta(S)$. As it is well-known, Smooth Ambiguity preferences satisfy Monotonicity, while in general they violate Certainty Independence. Assume now that $E \in$ $\Sigma$ satisfies $p(E)=q(E)=\rho \in(0,1)$ for all $p, q \in \operatorname{supp} \pi$. Then, $\succcurlyeq$ is $E$-biseparable, as for all $x \succcurlyeq y$,

$$
V(x E y)=\rho u(x)+(1-\rho) u(y)
$$

The existence of an event $E$ over which all the first-order beliefs agree is not a very strong con-

\footnotetext{
${ }^{20}$ A signed capacity is a set function $v: \Sigma \rightarrow \mathbb{R}$ such that $v(\varnothing)=0$ and $v(S)=1$. The non-monotonic Choquet integral is a Choquet integral (Choquet, 1954) with respect to a signed capacity $v$.
} 
dition. It may for instance be satisfied in the classical Ellsberg's 3-color urn experiment when $E$ denotes the event of a draw of a red ball from the urn. Thus, it is possible to provide a subjective mixture structure which may work with Smooth Ambiguity preferences. It is an open question whether it can be used to provide the model with a fully subjective axiomatic foundation.

Example 5. Consider a preference $\succcurlyeq$ satisfying the Vector Expected Utility model of Siniscalchi (2009). There is a Bernoulli utility $u$, a baseline prior $p$, an adjustment factor (a random variable) $\zeta$ with $\mathbb{E}_{p}(\zeta)=0,{ }^{21}$ and a function $A: \mathbb{R} \rightarrow \mathbb{R}$, with $A(0)=0$ and $A(r)=A(-r)$ for all $r \in \mathbb{R}$ such that $\succcurlyeq$ is represented by

$$
V(f)=\mathbb{E}_{p}[u \circ f]+A\left(\mathbb{E}_{p}[\zeta \cdot u \circ f]\right)
$$

As it is well-known, VEU preferences satisfy Monotonicity, while in general they violate Certainty Independence.

Assume that for an event $E \in \Sigma, p(E)=\frac{1}{2}, \zeta(s)=\frac{1}{2}$ for all $s \in E,-\zeta(s)=\frac{1}{2}$ for all $s \in E^{c}$, and $A(r)=-|r|$. Then, $\succcurlyeq$ is $E$-biseparable. Indeed, for all $x \succcurlyeq y$ it can be shown that ${ }^{22}$

$$
V(x E y)=\frac{1}{4} u(x)+\frac{3}{4} u(y)
$$

Example 6. Consider a preference $\succcurlyeq$ belonging to the class of Variational preferences of Maccheroni et al. (2006). There is a Bernoulli utility $u$, a cost function $c: \Delta(S) \rightarrow[0, \infty]$ such that $\succcurlyeq$ is represented by

$$
V(f)=\min _{p \in \Delta(S)} \mathbb{E}_{p}[u \circ f]+c(p)
$$

Suppose that there exists an event $E \in \Sigma$ such that $p(E)=q(E)=\rho \in(0,1)$ for all $p, q \in C$ where $C=\left\{r \in \Delta(S): c^{*}(r)=0\right\}$ and $c^{*}(r)=\sup _{f \in \mathscr{F}}\left(u\left(c_{f}\right)-\int_{S} u \circ f d r\right)$. Then, $\succcurlyeq$ is $E$-biseparable, as for all $x \succcurlyeq y$,

$$
V(x E y)=\rho u(x)+(1-\rho) u(y)
$$

\footnotetext{
${ }^{21}$ For simplicity we assume a single adjustment factor, but the model allows for any finite number of adjustment factors (see Siniscalchi, 2009).

${ }^{22}$ Since $V(x E y)=\frac{1}{2} u(x)+\frac{1}{2} u(y)-\left|\frac{1}{2} \frac{1}{2} u(x)-\frac{1}{2} \frac{1}{2} u(y)\right|$, when $x \succcurlyeq y, \frac{1}{4} u(x)-\frac{1}{4} u(y) \geq 0$, so that $V(x E y)=\frac{1}{4} u(x)+\frac{3}{4} u(y)$.
} 


\section{Extensions and discussion}

\subsection{Modeling "ex-ante" randomization}

Subjective mixtures of acts are defined state-by-state. In the traditional environment envisioned by AA, state-by-state mixtures are interpreted as follows: it is as if the DM has decided to commit to a certain randomization over the acts, which is performed after the revelation of the state of nature $s$. For this reason, it is customary to call them "ex-post" mixtures. AA also considered the possibility of "ex-ante" randomizations, which correspond to the more traditional idea of performing the randomization over the acts before the revelation of $s$. They also imposed an axiom, called "Reversal of Order in Compound Lotteries," which forces indifference between "ex-ante" and "ex-post" mixtures.

An obvious question is whether a similar distinction may be made in our subjective setting; i.e., how to provide a subjective notion of act mixture corresponding to AA's "ex-ante" randomizations. ${ }^{23}$ In the traditional AA setting with objective lotteries, when the DM performs an "ex-ante" 1/2:1/2 randomization between acts $f$ and $g$, she ends up choosing either $f$ or $g$, and subjecting herself to all the variability (over $S$ ) entailed by that act. It is as if she envisions receiving either $c_{f}$ or $c_{g}$ with probability $1 / 2$, as $c_{f}$ (respectively $c_{g}$ ) captures the DM's global evaluation of the act $f$ (respectively $g$ ). That is, the (objective) mixture $\frac{1}{2} c_{f}+\frac{1}{2} c_{g}$ is indifferent to the "ex-ante" mixture.

This intuition remains valid in our subjective setting. ${ }^{24}$ Given $f, g \in \mathscr{F}$, we call the "ex-ante" 1/2:1/2 subjective mixture of $f$ and $g$ a prize $z \in X$ such that $z=\frac{1}{2} c_{f} \oplus \frac{1}{2} c_{g}$, with $c_{f}$ (resp. $c_{g}$ ) a certainty equivalent of $f$ (resp. g). Given the representation functional $V$ obtained in Proposition 2 (and its restriction $u$ ), the utility of $z$ is

$$
V(z)=u(z)=\frac{1}{2} u\left(c_{f}\right)+\frac{1}{2} u\left(c_{g}\right)=\frac{1}{2} V(f)+\frac{1}{2} V(g)
$$

a 1/2:1/2 mixture of the "ex-ante" utilities of $f$ and $g$. We use $\oplus_{A}$ to denote this "ex-ante" mixture

\footnotetext{
${ }^{23}$ While in this section we use temporal locutions like "ex-ante," "ex-post" and "timing" for the sake of brevity, it is important to stress that in our setting this terminology is purely suggestive. Our subjective mixtures do not actually involve uncertainty and its resolution: $\frac{1}{2} f \oplus \frac{1}{2} g$ is an act which in state $s$ pays the certain prize $\frac{1}{2} f(s) \oplus \frac{1}{2} g(s)$; there is no uncertainty further to the state $s$. Hence time does not really play a role.

${ }^{24}$ Our proposal could also be given a more formal justification, based on a product state space setting, which is omitted for brevity.
} 
operation. That is, we set for all $\lambda \in[0,1]$ and all $f, g \in \mathscr{F}$,

$$
\lambda f \oplus_{A}(1-\lambda) g \equiv \lambda c_{f} \oplus(1-\lambda) c_{g}
$$

The mixture $\frac{1}{2} f \oplus_{A} \frac{1}{2} g$ is not necessarily indifferent to the "ex-post" subjective mixture $\frac{1}{2} f \oplus \frac{1}{2} g$. The comparison of $\frac{1}{2} f \oplus_{A} \frac{1}{2} g$ and $\frac{1}{2} f \oplus \frac{1}{2} g$ reflects a DM's difference in attitudes with respect to "exante" or "ex-post" randomizations. ${ }^{25}$ The following axiom spells out three possible attitudes with respect to the "timing" of mixtures:

Axiom. For all $f, g \in \mathscr{F}$ and all $\lambda \in[0,1]$ :

(a) (Preference for Ex-ante Mixtures - PEM) $\lambda f \oplus_{A}(1-\lambda) g \succcurlyeq \lambda f \oplus(1-\lambda) g$

(b) (Aversion to Ex-ante Mixtures - AEM) $\lambda f \oplus_{A}(1-\lambda) g \preccurlyeq \lambda f \oplus(1-\lambda) g$

(c) (Mixture Timing Indifference - MTI) $\lambda f \oplus_{A}(1-\lambda) g \sim \lambda f \oplus(1-\lambda) g$

Axiom MTI is the counterpart in our setting of AA's Reversal of Order axiom (but see the discussion in Supp. App. ??). It stipulates that for the DM it is equivalent to evaluate the act "ex-post" as a state-by-state mixture of $f$ and $g$, or "ex-ante" by evaluating $f$ and $g$ in isolation and then taking the corresponding subjective mixture of their certainty equivalents. In turn, Axioms PEM and AEM identify DMs whose preferences are sensitive to "timing" in a consistent fashion.

The following simple result shows that these properties translate immediately into properties of the representation functional $V$.

Proposition 3. Given a binary relation $\succcurlyeq$ with a locally biseparable representation $V$, it satisfies Axiom MTI (resp. PEM, AEM) if and only if $V$ is also affine (resp. convex, concave) with respect to $\oplus$.

Notice that this result does not require Monotonicity. It also implies that Axiom MTI is (in the presence of the other axioms) equivalent to the straightforward adaptation to subjective mixtures of the standard independence axiom (see Supp. App. ?? for details).

Thus, Axiom MTI precludes non-neutral attitudes toward ambiguity. More generally, imposing restrictions to the preference for the "timing" of randomization (PEM, AEM and MTI) imposes restrictions on "static" attitudes. For instance, Axiom AEM implies Axiom AH; i.e., a strong form of aversion to ambiguity. ${ }^{26}$

\footnotetext{
${ }^{25}$ See also Saito (2015) and Ke and Zhang (2018), which can be adapted to our subjective setting.

${ }^{26}$ These restrictions are clearly not embedded in our analysis. Other restrictions may be. For instance, Ke and Zhang (2018) introduce an axiom called "Indifference to Mixture Timing of Constant Acts," which our definition of "ex-ante" subjective mixture automatically guarantees.
} 
As discussed by Saito (2015) (see also Ke and Zhang (2018)), non-indifference to the subjective “timing” of uncertainty provides an answer to Raiffa (1961)'s normative criticism to ambiguity aversion. Raiffa suggests that the DM could neutralize the presence of ambiguity by performing an "ex-ante" randomization over two ambiguous acts, but he is assuming Reversal of Order (or MTI). If MTI is not satisfied, an "ex-ante" mixture will not hedge ambiguity as an "ex-post" mixture, and so it may not be more desirable than the ambiguous acts. For instance, if the DM satisfies Axiom AEM she could strictly prefer the "ex-post" mixture of the two ambiguous acts to either one of them, and instead be indifferent between the "ex-ante" mixture and the ambiguous acts.

\subsection{Subjective and objective mixtures}

As it is well-known, Schmeidler (1989), working in a traditional AA framework with an "objective" mixture notion, proposed the following definition of ambiguity aversion:

Axiom (Uncertainty Aversion - UA). For all $f, g \in \mathscr{F}, f \sim g \Longrightarrow \frac{1}{2} f+\frac{1}{2} g \succcurlyeq g$.

Here, $\frac{1}{2} f+\frac{1}{2} g$ denotes a $1 / 2: 1 / 2$ objective mixture of the acts $f$ and $g$. Thus, the natural interpretation of the UA axiom is a preference for objective randomization. ${ }^{27}$ However, there is increasing evidence that challenges the identification of preference for randomization and ambiguity aversion; e.g., Dominiak and Schnedler (2011). ${ }^{28}$

Our subjective mixture structure can help shed more light on this issue, by providing a separate identification of ambiguity aversion and preference for randomization. Indeed, subjective mixtures can be defined even when the set $X$ is equipped with an objective mixture structure. (For instance, when outcomes are monetary, or when they are objective lotteries over a set of prizes $Z$.) The midpoints obtained via $\oplus$ and the objective mixture + may then be different. Two observations follow from this discrepancy. First, it can be exploited to model preferences which display both the Allais and Ellsberg modal choices, as in Dean and Ortoleva (2017). ${ }^{29}$ Second, it implies

\footnotetext{
${ }^{27}$ Indeed, Schmeidler writes (p.582) that “[...] substituting objective mixing for subjective mixing makes the decision maker better off," where by "subjective mixing” he means eventwise mixing such as that accomplished by constructing the act $f A g$ for some event $A$, and not our subjective mixtures.

${ }^{28}$ See also the discussion in Ke and Zhang (2018) and the references cited therein.

${ }^{29}$ Since by construction the utility $u$ is affine with respect to $\oplus$, we have the immediate observation that $\frac{1}{2} x+\frac{1}{2} y \succcurlyeq$ (resp. $\preccurlyeq) \frac{1}{2} x \oplus \frac{1}{2} y$ for all $x \succcurlyeq y$ if and only if $u$ is concave (resp. convex) with respect to + . If $X=\Delta(Z)$, with + representing probability mixture, strict concavity or convexity imply non-linear utility over lotteries. Our construction allows for a wider spectrum of attitudes with respect to ambiguity than Dean and Ortoleva (2017), as we do not impose any additional independence assumption (such as their Degenerate Independence axiom).
} 
that the UA axiom and the $\mathrm{AH}$ axiom (see Remark 2) are independent properties. For instance, a DM could display, for given $f \sim g$, either

$$
\frac{1}{2} f+\frac{1}{2} g>f \sim \frac{1}{2} f \oplus \frac{1}{2} g
$$

or

$$
\frac{1}{2} f \oplus \frac{1}{2} g>f \sim \frac{1}{2} f+\frac{1}{2} g
$$

Eq. (8) is compatible with a DM who is ambiguity (hedging) neutral and displays a preference for objective randomization. For example, assume that outcomes are objective lotteries over an interval $Z \subseteq \mathbb{R}$ (i.e., $X=\Delta(Z)$ ) and consider the preference $\succcurlyeq$ represented by $V(f)=\mathbb{E}_{p}[u \circ f]$, for some $p \in \Delta(S)$, where $u$ is a Cautious EU in the sense of Cerreia-Vioglio, Dillenberger, and Ortoleva (2015): for every $s \in S$ and $f \in \mathscr{F}, u(f(s))=\min _{\nu \in \mathcal{V}} v^{-1}\left(\mathbb{E}_{f(s)}[v]\right)$. This preference is easily seen to satisfy Eq. (8) for all $f$ and $g$. In contrast, Eq. (9) is compatible with a DM who displays ambiguity hedging while being neutral with respect to the objective randomization. Supplementary Appendix ?? provides an example of such a DM, as well as another example of a preference satisfying Eq. (8).

The discrepancy is also relevant for experimental design. Recently, there has been some discussion about whether a preference for randomization could undermine the incentive compatibility of the random incentive system (RIS), a common payment scheme used in laboratory experiments on ambiguity aversion (e.g., Bade, 2015; Baillon, Halevy, and Li, 2015; Azrieli, Chambers, and Healy, 2018). The RIS pays randomly one of the choices made by the subject during the experiment; it could therefore play the role of an objective randomization device, thus affecting the truthful revelation of "ambiguity aversion." 30 However, this eventuality is based on the identification of preference for randomization and ambiguity aversion. By severing the tie between ambiguity aversion and preference for randomization, our analysis suggests that the RIS is not necessarily affected by subjects' ambiguity reaction. That is, the focus of the discussion on the appropriateness of RIS should be on the empirical relevance of preference for randomization, rather than ambiguity aversion.

\footnotetext{
${ }^{30}$ The argument is identical to Raiffa's critique. By randomizing the DM can create a lottery over payoffs that hedges ambiguity.
} 


\section{Appendices}

\section{A Local biseparability and SOSEU}

The examples in Section 4 show that local biseparability allows a wide range of preference models. However, there is a class of preferences which are not in general locally biseparable: the secondorder subjective expected utility (SOSEU) preferences of Grant et al. (2009). In this model $\succcurlyeq$ is represented by

$$
V(f)=\phi^{-1}\left(\int_{S} \phi(u \circ f) d p\right)
$$

for some monotone function $\phi: u(X) \rightarrow \mathbb{R}$ and a cardinally unique $u: X \rightarrow \mathbb{R}$. The SEU model corresponds to a linear $\phi$. It is well-known that, in Savage's setting, SEU and SOSEU are observationally indistinguishable (see, for example, Strzalecki, 2011). This is because it is then difficult to separately identify $u$ and $\phi$.

Subjective mixtures might provide a way to circumvent this impossibility, as they allow to identify $u$. However, any SOSEU preference which is locally biseparable must have an affine $\phi$ and hence be SEU. ${ }^{31}$

Fact 1. Given a SOSEU preference $\succcurlyeq, E \in \Sigma$ satisfies ordered bisymmetry if and only if it $\succcurlyeq$ is SEU (i.e. $\phi(k)=a k+b$, for some $a>0, b \in \mathbb{R})$.

This fact leverages on the following simple mathematical result:

Lemma 2. If a function $\phi:[a, b] \rightarrow \mathbb{R}$ is not affine, for all $p \in(0,1)$, there are at least two real numbers $r \neq s \in[a, b]$ such that $\phi(p r+(1-p) s) \neq p \phi(r)+(1-p) \phi(s)$.

Proof. Suppose not, then for all $r, s \in[a, b], \phi(p r+(1-p) s)=p \phi(r)+(1-p) \phi(s)$, the fact that $p \in(0,1)$ allows to apply Aczél (1966, Th. 1, p. 66) and conclude that $\phi(r)=\alpha r+\beta$ for some $\alpha \neq 0$, $\beta \in \mathbb{R}$, a contradiction to the assumption that $\phi$ is not affine.

Proof of Fact 1. Consider a SOSEU preference with a non-affine $\phi$. By Lemma 2, for all $p \in(0,1)$ there are $r, s \in u(X)$ with $\phi(p r+(1-p) s) \neq p \phi(r)+(1-p) \phi(s)$. W.l.o.g. let $r>s$ and take $x, z, z^{\prime}, y \in X$

\footnotetext{
${ }^{31}$ As Multiplier Preferences (Hansen and Sargent, 2001) lie in the intersection of the Variational Preference and SOSEU models (see Strzalecki, 2011), it follows that our setting cannot be used to characterize them behaviorally.
} 
with $u(x)=u(z)=r$ and $u(y)=u\left(z^{\prime}\right)=s$. By ordered bisymmetry:

$$
\begin{aligned}
V\left(c_{x E z} E c_{z^{\prime} E y}\right) & =\phi^{-1}\left(p \phi(p u(x)+(1-p) u(z))+(1-p) \phi\left(p u\left(z^{\prime}\right)+(1-p) u(y)\right)\right) \\
& =\phi^{-1}\left(p \phi\left(p u(x)+(1-p) u\left(z^{\prime}\right)\right)+(1-p) \phi(p u(z)+(1-p) u(y))\right) \\
& =V\left(c_{x E z^{\prime}} E c_{z E y}\right)
\end{aligned}
$$

The definitions of $u(x), u(y), u(z), u\left(z^{\prime}\right)$ and the second equality above imply the contradiction:

$$
p \phi(r)+(1-p) \phi(s)=p \phi(p r+(1-p) s)+(1-p) \phi(p r+(1-p) s)=\phi(p r+(1-p) s)
$$

\section{B Consistent biseparability}

Here we present a preference restriction that, leveraging on Lemma 1, guarantees that the mixture space structure does not depend on the $(\mathrm{ON})$ event used to construct it. Start by defining $\mathscr{E} \equiv$ $\{E \in \Sigma: E$ satisfies $O N\}$; i.e., the events which may be used to obtain a biseparable representation. Consider now the following preference restriction:

Axiom (Consistent Biseparability - CB). Given any $E, F \in \mathscr{E}$, they are average-equivalent.

As its name suggests, this axiom guarantees the equivalence of utility midpoints across different (biseparable) representations of $\succcurlyeq$, and thus that the preference's biseparability is consistent. It follows from the discussion at the end of Section 3.1 that Axiom CB is weaker than the assumptions made in GMMS.

Axiom CB allows us to add structure to the representation in Proposition 2:

Proposition 4. $\succcurlyeq$ is a binary relation satisfying Axioms $P, C, C B$, and there exists an ON event $E \in \Sigma$ if and only if there exist a nontrivial, constant-bounded and subcontinuous functional $V: \mathscr{F} \rightarrow \mathbb{R}$ which represents $\succcurlyeq$, and a nonempty set $\mathscr{E} \subseteq \Sigma$ and a function $\rho: \mathscr{E} \rightarrow(0,1)$ such that $F \in \mathscr{E}$ if and only iffor all $x \succcurlyeq y$

$$
V(x F y)=\rho(F) u(x)+(1-\rho(F)) u(y)
$$

Moreover, $V$ is unique up to positive affine transformations and $\rho$ and $\mathscr{E}$ are unique.

Proof. For sufficiency, let $\mathscr{E}$ be as defined above Axiom CB, it follows from Proposition 2 that $\succcurlyeq$ is $E$-biseparable for every $E \in \mathscr{E}$. If there is a unique $E \in \mathscr{E}$, it is sufficient to define $\rho_{E}=\rho(E)$ 
and $u=u_{E}$. Assume then the existence of $F \in \mathscr{E}$ with $F \neq E$. Take $x \succcurlyeq y$ and consider $V_{E}(x E y)=$ $\rho_{E} u_{E}(x)+\left(1-\rho_{E}\right) u_{E}(y)$ and $V_{F}(x E y)$. By Lemma 1, Axioms P, C, and CB imply that $u_{E}$ and $u_{F}$ (resp $V_{E}$ and $V_{F}$ ) are cardinally equivalent, hence we can normalize them to be equal: $u=u_{E}=u_{F}$ $\left(\right.$ resp $\left.V_{E}=V_{F}=V\right)$. Therefore, for any $x \succcurlyeq y, V(x E y)=V_{F}(x E y)=u(x) \rho_{E}+\left(1-\rho_{E}\right) u(y)$. Defining $\rho: \mathscr{E} \rightarrow(0,1)$ by $\rho(E) \equiv \rho_{E}$ concludes the proof. The cardinality of $V$ follows from Def. 2 . The uniqueness of $\mathscr{E}$ is immediate, and it implies, alongside Def. 2, the uniqueness of $\rho$.

To see necessity, Axioms $\mathrm{P}$ and $\mathrm{C}$ follow from the properties of $V$. For CB, given the set $\mathscr{E} \neq \varnothing$ specified in the statement, consider $E, F \in \mathscr{E}$. It follows from Eq. (10) and Proposition 2 that $\succcurlyeq$ is $E$ and $F$-biseparable, and by the uniqueness properties of the biseparable representation it follows that $u_{F} \approx u \approx u_{E}$. But then $V_{E}$ and $V_{F}$ are cardinally equivalent and so by Lemma $1, E$ and $F$ are average-equivalent and hence CB holds on $\mathscr{E}$.

Three comments on this representation are in order. First, it shows that the Axioms P, C and CB make it possible to tell apart —in fully subjective environments— differences in traditional risk attitude (as represented by the behavior of $u$ ) and other subjective traits like ambiguity aversion (see the discussion in Ghirardato and Marinacci (2002)). Second, without imposing Monotonicity it may not be the case that the set-function $\rho$ is itself monotonic: given $E, F \in \mathscr{E}$, it is not necessarily true that $E \subseteq F$ implies $\rho(E) \leq \rho(F)$. Third, even if both $E$ and $E^{c}$ belong to $\mathscr{E}$, it may not be the case that $\rho(E)+\rho\left(E^{c}\right)=1$ (see Example 2 in Section 4). That is, the preference over bets on $E$ or $E^{c}$ is not necessarily represented by expected utility.

\section{Proofs}

Proof of Proposition 1. We only prove the existence of $E$-preference averages, since the fact that $E$-preference averages are equivalent to utility midpoints is straightforward; see GMMSa. Since $\succcurlyeq$ is $E$-biseparable, $u=V_{\mid X}$ is $\tau$-continuous. Therefore, for all $x, y \in X$ with $x \succcurlyeq y$, there is $z \in X$ such that $u(z)=\frac{1}{2} u(x)+\frac{1}{2} u(y)$. By $E$-biseparability again, $V\left(c_{x E z} E c_{z E y}\right)=\rho_{E}\left(\rho_{E} u(x)+\left(1-\rho_{E}\right) u(z)\right)+$ $\left(1-\rho_{E}\right)\left(\rho_{E} u(z)+\left(1-\rho_{E}\right) u(y)\right)=\rho_{E} u(x)+\left(1-\rho_{E}\right) u(y)=V(x E y)$, hence $z$ is an $E$-preference average of $x, y \in X$.

Lemma 3. Suppose that $\succcurlyeq$ satisfies $P$ and $C$. Then for any $f \in \mathscr{F}$, there exists $c_{f} \in X$ such that $c_{f} \sim f$.

Proof. By P, for each $f \in \mathscr{F}$, there are $x, y \in X$ with $x \succcurlyeq f \succcurlyeq y$, hence $B=\{z \in X: z \succcurlyeq f\}$ and $W=$ 
$\{z \in X: f \succcurlyeq z\}$ are both non-empty. By C both sets are closed, hence connectedness of $X$ implies non-emptiness of $B \cap W$. So, for all $f \in \mathscr{F}$ there exists $c_{f} \in X$ with $c_{f} \sim f$.

Proof of Proposition 2. To prove sufficiency, we show that our axioms implies axioms A1, A2, A3, A5 and A6 of Nakamura (1990) for the set s of the bets on E. P implies A1. A2 and A5 follow from $\mathrm{C}$ and the Structural Assumption. A3 is equal to betwise monotonicity. Axiom A6 is equivalent to ordered bisymmetry. Therefore, by Lemma 3 in Nakamura (1990, it can be seen from the proof that the result does not require his axiom A4), there exist a unique $\rho_{E} \in(0,1)$ and a cardinally unique function $u: X \rightarrow \mathbb{R}$ such that the functional $V: \mathscr{F} \rightarrow \mathbb{R}$ defined by $V(f)=u\left(c_{f}\right)$ (certainty equivalents exist by Lemma 3) represents $\succcurlyeq$ and, for all $x \succcurlyeq y \in X, V(x E y)=\rho_{E} u(x)+\left(1-\rho_{E}\right) u(y)=$ $\rho_{E} V(x)+\left(1-\rho_{E}\right) V(y)$. Nontriviality and constant-boundedness of $V$ follow from Axiom P, while subcontinuity is shown as in Lemma 31 in Ghirardato and Marinacci (2001).

To show necessity, we first show that $E$ is ordered-bisymmetric: $V\left(c_{x E z} E c_{z^{\prime} E y}\right)=\rho_{E}\left(\rho_{E} V(x)+\right.$ $\left.\left.\left(1-\rho_{E}\right) V(z)\right)+\left(1-\rho_{E}\right)\left(\rho_{E} V\left(z^{\prime}\right)+\left(1-\rho_{E}\right) V(y)\right)=\rho_{E}^{2} V(x)+\rho_{E}\left(1-\rho_{E}\right) V(z)\right)+\left(1-\rho_{E}\right) \rho_{E} V\left(z^{\prime}\right)+(1-$ $\left.\rho_{E}\right)^{2} V(y)=\rho_{E}\left(\rho_{E} V(x)+\left(1-\rho_{E}\right) V\left(z^{\prime}\right)\right)+\left(1-\rho_{E}\right)\left(\rho_{E} V(z)+\left(1-\rho_{E}\right) V(y)\right)=V\left(c_{x E z^{\prime}} E c_{z E y}\right)$. The fact that $\rho_{E} \in(0,1)$ implies immediately that $E$ is betwise-monotonic, hence $E$ is ordered-nice. The necessity of Axioms $\mathrm{P}$ and $\mathrm{C}$ follows immediately from the properties of $V$.

Proof of Lemma 1. Take $x, y \in X$ with $x \succcurlyeq y$, and two events $E, F \in \Sigma^{\prime}$ such that $\succcurlyeq$ is $E$ - and $F$ biseparable. Then, there exist two cardinally unique representations of $\succcurlyeq, V_{E}: X \rightarrow \mathbb{R}$ and $V_{F}$ : $X \rightarrow \mathbb{R}$. Since $V_{E}$ and $V_{F}$ represent the same preferences over $X$, there exists a monotone and continuous function $\phi: V_{F}(X) \rightarrow V_{E}(X)$ such that $V_{E}=\phi\left(V_{F}\right)$. Suppose that $V_{E}(z)=\frac{1}{2} V_{E}(x)+\frac{1}{2} V_{E}(y)$ and $V_{F}\left(z^{\prime}\right)=\frac{1}{2} V_{F}(x)+\frac{1}{2} V_{F}(y)$ imply $V_{E}(z)=V_{E}\left(z^{\prime}\right)$ and $V_{F}(z)=V_{F}\left(z^{\prime}\right)$. Then, $\phi\left(V_{F}\left(z^{\prime}\right)\right)=V_{E}(z)=$ $\frac{1}{2} \phi\left(V_{F}(x)\right)+\frac{1}{2} \phi\left(V_{F}(y)\right)$ but also $\phi\left(V_{F}\left(z^{\prime}\right)\right)=\phi\left(\frac{1}{2} V_{F}(x)+\frac{1}{2} V_{F}(y)\right)$. The continuous solution to the standard Jensen's functional equation $\frac{1}{2} \phi(r)+\frac{1}{2} \phi(s)=\phi\left(\frac{1}{2} r+\frac{1}{2} s\right)$ is $\phi(r)=\alpha r+\beta$ (Aczél, 1966), for $\alpha \neq 0, \beta \in \mathbb{R}$. That is, $V_{E}=\alpha V_{F}+\beta$, with $\alpha>0$ since $V_{F}$ and $V_{E}$ represent the same preference. For the opposite direction, suppose that $\succcurlyeq$ is $E$ - and $F$-biseparable with representations $V_{E}$ and $V_{F}$ respectively. Proposition 1 and the indifferences $x E y \sim c_{x E z} E c_{z E y}$ and $x E y \sim c_{x E z^{\prime}} E c_{z E y^{\prime}}$ are equivalent to $V_{E}(z)=\frac{1}{2} V_{E}(x)+\frac{1}{2} V_{E}(y)$ and $V_{F}\left(z^{\prime}\right)=\frac{1}{2} V_{F}(x)+\frac{1}{2} V_{F}(y)$. Since $V_{E} \approx V_{F}$, there are $\alpha>0$ and $\beta \in \mathbb{R}$ such that $V_{F}=\alpha V_{E}+\beta$. Therefore, $V_{F}\left(z^{\prime}\right)=\frac{1}{2} \alpha V_{E}(x)+\frac{1}{2} \alpha V_{E}(y)+\beta=\alpha\left[\frac{1}{2} V_{E}(x)+\frac{1}{2} V_{E}(y)\right]+\beta=$ $\alpha V_{E}(z)+\beta=V_{F}(z)$ implying $z \sim z^{\prime}$ so that $E$ and $F$ are average-equivalent.

Proof of Proposition 3. Assume that $\succcurlyeq$ satisfies the assumptions of Proposition 2 and let $u(x)=$ 
$V(x)$ for all $x \in X$, so that Eq. (4) holds. By Eq. (4) and Axiom PEM, $\lambda V(f)+(1-\lambda) V(g)=\lambda u\left(c_{f}\right)+$ $(1-\lambda) u\left(c_{g}\right)=u\left(\lambda c_{f} \oplus(1-\lambda) c_{g}\right)=V\left(\lambda c_{f} \oplus(1-\lambda) c_{g}\right) \geq V(\lambda f \oplus(1-\lambda) g)$ for any $\lambda \in[0,1]$. Hence $V$ is convex w.r.t. $\oplus$. The opposite implication follows by reversing the argument. The proofs that AEM is equivalent to convexity and MTI to affinity of $V$ are analogous.

\section{References}

Joseph Aczél. Lectures on functional equations and their applications, volume 19. Academic Press, 1966.

Shiri Alon and David Schmeidler. Purely subjective maxmin expected utility. Journal of Economic Theory, 152:382-412, 2014.

Francis J Anscombe and Robert J Aumann. A definition of subjective probability. The Annals of Mathematical Statistics, 34(1):199-205, 1963.

Yaron Azrieli, Christopher P Chambers, and Paul J Healy. Incentives in experiments: A theoretical analysis. Journal of Political Economy, 126(4):1472-1503, 2018.

Sophie Bade. Randomization devices and the elicitation of ambiguity-averse preferences. Journal of Economic Theory, 159:221-235, 2015.

Aurélien Baillon and Lætitia Placido. Testing constant absolute and relative ambiguity aversion. Journal of Economic Theory, 181:309 - 332, 2019.

Aurélien Baillon, Yoram Halevy, and Chen Li. Experimental elicitation of ambiguity attitude using the random incentive system. Mimeo, 2015.

Truman F Bewley. Knightian decision theory. Part I. Decisions in Economics and Finance, 25(2): 79-110, 2002.

Antoine Bommier. A dual approach to ambiguity aversion. Journal of Mathematical Economics, $71: 104-118,2017$.

Ramon Casadesus-Masanell, Peter K Klibanoff, and Emre Ozdenoren. Maxmin expected utility over Savage acts with a set of priors. Journal of Economic Theory, 92(1):35-65, 2000.

Simone Cerreia-Vioglio, Paolo Ghirardato, Fabio Maccheroni, Massimo Marinacci, and Marciano Siniscalchi. Rational preferences under ambiguity. Economic Theory, 48(2):341-375, 2011a. 
Simone Cerreia-Vioglio, Fabio Maccheroni, Massimo Marinacci, and Luigi Montrucchio. Uncertainty averse preferences. Journal of Economic Theory, 146(4):1275-1330, $2011 \mathrm{~b}$.

Simone Cerreia-Vioglio, David Dillenberger, and Pietro Ortoleva. Cautious expected utility and the certainty effect. Econometrica, 83(2):693-728, 2015.

Alain Chateauneuf and Josè Heleno Faro. Ambiguity through confidence functions. Journal of Mathematical Economics, 45(9-10):535-558, 2009.

Patrick Cheridito, Freddy Delbaen, Samuel Drapeau, and Michael Kupper. Numerical representation of convex preferences over Anscombe-Aumann acts. Mimeo, 2015.

Gustave Choquet. Theory of capacities. Annales de l'Institut Fourier, 5:131-295, 1954.

Eric Danan, Thibault Gajdos, Brian Hill, and Jean-Marc Tallon. Robust social decisions. American Economic Review, 106(9), 2016.

Anja De Waegenaere and Peter P Wakker. Nonmonotonic Choquet integrals. Journal of Mathematical Economics, 36(1):45-60, 2001.

Mark Dean and Pietro Ortoleva. Allais, Ellsberg, and preferences for hedging. Theoretical Economics, 12(1):377-424, 2017.

Eddie Dekel, Barton L Lipman, and Aldo Rustichini. Temptation-driven preferences. The Review of Economic Studies, 76(3):937-971, 2009.

Adam Dominiak and Wendelin Schnedler. Attitudes toward uncertainty and randomization: an experimental study. Economic Theory, 48(2-3):289-312, 2011.

Jürgen Eichberger and David Kelsey. Uncertainty aversion and preference for randomisation. Journal of Economic Theory, 71(1):31 - 43, 1996.

Andrew Ellis and Michele Piccione. Correlation misperception in choice. American Economic Review, 107(4):1264-92, 2017.

Peter C Fishburn. Utility theory for decision making. Wiley, New York, 1970.

Paolo Ghirardato and Massimo Marinacci. Risk, ambiguity, and the separation of utility and beliefs. Mathematics of Operations Research, 26(4):864-890, 2001.

Paolo Ghirardato and Massimo Marinacci. Ambiguity made precise: A comparative foundation. Journal of Economic Theory, 102(2):251-289, 2002. 
Paolo Ghirardato and Daniele Pennesi. Mixing without randomness. Carlo Alberto Notebook 593, Collegio Carlo Alberto, 2019.

Paolo Ghirardato, Fabio Maccheroni, Massimo Marinacci, and Marciano Siniscalchi. A subjective spin on roulette wheels. Working Paper 1127, California Institute of Technology, Division of H\&SS, 2001.

Paolo Ghirardato, Fabio Maccheroni, Massimo Marinacci, and Marciano Siniscalchi. A subjective spin on roulette wheels. Working Paper 1127R, California Institute of Technology, Division of H\&SS, 2002.

Paolo Ghirardato, Fabio Maccheroni, Massimo Marinacci, and Marciano Siniscalchi. A subjective spin on roulette wheels. Econometrica, 71(6):1897-1908, 2003.

Paolo Ghirardato, Fabio Maccheroni, and Massimo Marinacci. Differentiating ambiguity and ambiguity attitude. Journal of Economic Theory, 118(2):133-173, 2004.

Itzhak Gilboa. Expected utility with purely subjective non-additive probabilities. Journal of Mathematical Economics, 16(1):65-88, 1987.

Itzhak Gilboa. Expectation and variation in multi-period decisions. Econometrica, 56(5):11531169, 1989.

Itzhak Gilboa and David Schmeidler. Maxmin expected utility with non-unique prior. Journal of Mathematical Economics, 18(2):141-153, 1989.

Simon Grant and Atsushi Kajii. The epsilon-Gini-contamination multiple priors model admits a linear-mean-standard-deviation utility representation. Economics Letters, 95(1):39-47, 2007.

Simon Grant and Ben Polak. Mean-dispersion preferences and constant absolute uncertainty aversion. Journal of Economic Theory, 148(4):1361 - 1398, 2013.

Simon Grant, Ben Polak, and Tomasz Strzalecki. Second-order expected utility. Mimeo, 2009.

Faruk Gul and Wolfgang Pesendorfer. Temptation and self-control. Econometrica, 69(6):14031435, 2001.

Lars Peter Hansen and Thomas J Sargent. Robust control and model uncertainty. American Economic Review, 91(2):60-66, 2001.

Shaowei Ke and Qu Zhang. Randomization and ambiguity aversion. Mimeo, 2018. 
Peter Klibanoff, Sujoy Mukerji, and Kyoungwon Seo. Perceived ambiguity and relevant measures. Econometrica, 82(5):1945-1978, 2014.

Peter K Klibanoff, Massimo Marinacci, and Sujoy Mukerji. A smooth model of decision making under ambiguity. Econometrica, 73(6):1849-1892, 2005.

Fabio Maccheroni, Massimo Marinacci, and Aldo Rustichini. Ambiguity aversion, robustness, and the variational representation of preferences. Econometrica, 74(6):1447-1498, 2006.

Yutaka Nakamura. Subjective expected utility with non-additive probabilities on finite state spaces. Journal of Economic Theory, 51(2):346-366, 1990.

Howard Raiffa. Risk, ambiguity, and the Savage axioms: comment. The Quarterly Journal of Economics, 75(4):690-694, 1961.

Kota Saito. Preferences for flexibility and randomization under uncertainty. American Economic Review, 105(3):1246-71, 2015.

Leonard J. Savage. The Foundations of Statistics. Wiley, New York, 1954.

David Schmeidler. Subjective probability and expected utility without additivity. Econometrica, 57(3):571-587, 1989.

Florian Schneider and Martin Schonger. An experimental test of the Anscombe-Aumann Monotonicity axiom. Working Paper 207, Department of Economics, University of Zurich, 2015.

Kyoungwon Seo. Ambiguity and second-order belief. Econometrica, 77(5):1575-1605, 2009.

Marciano Siniscalchi. Vector expected utility and attitudes toward variation. Econometrica, 77(3): 801-855, 2009.

Tomasz Strzalecki. Axiomatic foundations of multiplier preferences. Econometrica, 79(1):47-73, 2011.

John von Neumann. Various techniques used in connection with random digits. National Bureau of Standards Applied Mathematics Series, 12:36-38, 1951. 


\title{
A general theory of subjective mixtures (Supplementary appendices)
}

\author{
Paolo Ghirardato $^{\S}$ Daniele Pennesi ${ }^{\llbracket}$ \\ December 2019 \\ This version: March 2020
}

\section{Supplementary appendices}

\section{D.1 Global properties}

In this Appendix, we show the effects of imposing some global behavioral restrictions to locally biseparable preferences. We start by considering a mild condition, called "decomposability," and then move on to consider the effects of adding full Monotonicity, a weak type of Certainty Independence, and finally a type of ambiguity aversion.

\section{D.1.1 Further preliminaries}

We denote by $B_{0}(\Sigma, \Gamma)$ the set of simple $\Sigma$-measurable functions on $S$ taking value in $\Gamma \subseteq \mathbb{R}$. A functional $I: B_{0}(\Sigma, \Gamma) \rightarrow \mathbb{R}$ is said to be: monotonic if $I(a) \geq I(b)$ when $a \geq b$, continuous if it is sup-norm continuous, normalized if $I\left(\gamma 1_{S}\right)=\gamma$ for all $\gamma \in \Gamma$. I is said to be convex (resp. concave) if, for all $a, b \in B_{0}(\Sigma, \Gamma)$ and all $\lambda \in[0,1], I(\lambda a+(1-\lambda) b) \leq \lambda I(a)+(1-\lambda) I(b)($ resp. $I(\lambda a+(1-\lambda) b) \geq$ $\lambda I(a)+(1-\lambda) I(b))$, affine if it is both convex and concave. A set function $\rho: \Sigma \rightarrow[0,1]$ is called a capacity if it is normalized $(\rho(\varnothing)=0$ and $\rho(S)=1)$, and monotone $(\rho(A) \geq \rho(B)$ whenever $B \subseteq A)$. Finally, given $u: X \rightarrow \mathbb{R}$, we define $X_{u \uparrow}^{2}=\left\{(x, y) \in X^{2}: u(x) \geq u(y)\right\}$.

\section{D.1.2 Decomposable preferences}

Consider the following property, which stipulates that two acts be indifferent whenever they are indifferent state-by-state.

\footnotetext{
${ }^{\S}$ DESOMAS and Collegio Carlo Alberto, University of Torino, email: paolo.ghirardato@unito.it

'DESOMAS, University of Torino, email: daniele.pennesi@unito.it
} 
Axiom (Decomposability - D). For all $f, g \in \mathscr{F}, f(s) \sim g(s)$ for all $s \in S$ implies $f \sim g$.

This is a weak form of separability across states, implied by standard Monotonicity. It is called “indifference substitution” in Grant and Polak (2013). Decomposable but non-monotone preferences are the Mean-standard deviation preferences of example 2 and the Signed Choquet preferences of example 3.

Adding Decomposability to the axioms that characterize local biseparability allows us to rewrite the representation $V$ of $\succcurlyeq$ defined on $\mathscr{F}$ as the composition $V=I \circ u$ of the Bernoulli utility $u$ and a functional $I: B_{0}(\Sigma, u(X)) \rightarrow \mathbb{R}$ which describes the DM's handling of uncertainty. An act $f$ is then identified with its utility profile $u \circ f$ and a subjective mixture of acts $\frac{1}{2} f \oplus \frac{1}{2} g$ with the mixture of utility profiles $\frac{1}{2} u \circ f+\frac{1}{2} u \circ g$.

Proposition 5. $\succcurlyeq$ satisfies Axioms $P, C, D$, and there exists an $O N$ event $E \in \Sigma$ if and only if there is a representation $V$ of $\succcurlyeq$ that can be written as $V=I \circ u$, where $u: X \rightarrow \mathbb{R}$ is a $\tau$-continuous, nonconstant function, and $I: B_{0}(\Sigma, u(X)) \rightarrow \mathbb{R}$ is a normalized, continuous functional such that for all binary acts $x$ Ay with $x \succcurlyeq y$ and $A \in \Sigma$,

$$
I(u \circ(x A y))=\rho_{x, y}(A) u(x)+\left(1-\rho_{x, y}(A)\right) u(y)
$$

for some $\rho: X_{u \uparrow}^{2} \times \Sigma \rightarrow \mathbb{R}$ such that $\rho_{x, y}(E) \equiv \rho_{E} \in(0,1)$ for all $(x, y) \in X_{u \uparrow}^{2}$ and for some $E \in \Sigma$. ${ }^{1}$

Proof of Proposition 5. For sufficiency, by Corollary 1, P, C, and the existence of an event $E$ that satisfies ON imply the existence of a cardinally unique non-constant $u=\left.V\right|_{X}$ representing $\succcurlyeq$ on $X$. It is w.l.o.g. to choose $u$ s.t. $[-1,1] \subseteq u(X) \subseteq \mathbb{R}$. Denote by $B_{0}(\Sigma, u(X))$ the subset of $B_{0}(\Sigma)$ consisting of simple measurable functions with range in $u(X)$. It is simple to show that $B_{0}(\Sigma, u(X))=\{u \circ f: f \in \mathscr{F}\}$. Let $I(u \circ f)=u\left(c_{f}\right)$ for all $f \in \mathscr{F}$. Clearly, $u \circ f=u \circ g$, if and only if, $f(s) \sim g(s)$ for all $s \in S$, by Decomposability, $f \sim g$ and $I(u \circ f)=u\left(c_{f}\right)=u\left(c_{g}\right)=I(u \circ g)$. Hence $I: B_{0}(\Sigma, u(X)) \rightarrow \mathbb{R}$ is well defined. It is also normalized, pick $k \in u(X)$. By assumption, there exists $x \in X$ such that $u(x)=k$, letting $a=k 1_{S}$, then $a=u \circ f$ with $f=x, I(a)=u\left(c_{f}\right)=u(x)=k$. To prove continuity, consider the set $L \equiv\left\{a \in B_{0}(\Sigma, u(X)): I(a) \leq k\right\}$, and assume it is open. Then there exists a sequence $a_{n} \rightarrow a$, such that $I\left(a_{n}\right) \leq k$ but $I(a)>k$. It follows that there exists a net $f_{\alpha} \in \mathscr{F}$, with $a_{\alpha}=u \circ f_{\alpha} \rightarrow u \circ f=a$ with $I\left(u \circ f_{\alpha}\right)=u\left(c_{f_{\alpha}}\right) \leq u(z)$ where $I(u \circ z)=u(z)=k$ and $I(u \circ f)=u\left(c_{f}\right)>$

\footnotetext{
${ }^{1}$ As to uniqueness: $\rho(\cdot)$ is unique, while if $J \circ v$ also represents $\succcurlyeq$, with $v: X \rightarrow \mathbb{R}$ and $J: B_{0}(\Sigma, \nu(X)) \rightarrow \mathbb{R}$, there exist $\mu \in \mathbb{R}, \lambda>0$, such that, $v(x)=\lambda u(x)+\mu$, and $J(b)=\lambda I\left(\lambda^{-1}[b-\mu]\right)+\mu$ for all $b \in B_{0}(\Sigma, v(X))$.
} 
$u(z)$. Since $u$ represents preferences on $X, u\left(c_{f_{\alpha}}\right) \rightarrow u\left(c_{f}\right)$ and $u\left(c_{f_{\alpha}}\right) \leq u(z)<u\left(c_{f}\right)$ for all $\alpha$, contradicts $\tau$-continuity of $u$. Hence, $I$ is lower-semicontinuous. Upper-semicontinuity follows from a symmetric argument. So $I$ is continuous. Hence, the first part is proved.

For the statements on binary acts, notice first of all that for the ON event $E$, then by Proposition 2, $V(x E y)=I(u \circ(x E y))=\rho_{E} u(x)+\left(1-\rho_{E}\right) u(y)$, for some $\rho_{E} \in(0,1)$. So, for any $(x, y) \in X_{u \uparrow}^{2}$, let $\rho_{x, y}(E)=\rho_{E}$. Next for any $A \in \Sigma \backslash E$ and $(x, y) \in X_{u \uparrow}^{2}$, let $\rho_{x, y}(A) \equiv \frac{I(u \circ(x A y))-u(y)}{u(x)-u(y)}$ when $u(x)>u(y)$ and $\rho_{x, y}(A) \equiv \frac{1}{2}$ when $u(x)=u(y)$. Therefore, for all $A \in \Sigma$, Eq. (11) holds. The uniqueness part follows from the definition of locally biseparable representation.

The necessity of Axioms P, C and D is straightforward from the properties of $u$ and $I$. Since there is an event $E \in \Sigma$ such that $\rho_{x, y}(E)=\rho_{E} \in(0,1)$ for all $(x, y) \in X_{u \uparrow}^{2}$ and Eq. (11) holds, it follows that $\succcurlyeq$ is $E$-biseparable. By Prop. 2, E satisfies ON.

This representation generalizes the one given by CGMMS, as it shows that a Bernoullian and Archimedean representation of preferences can be obtained by (Decomposability and) a form of separability on a single event $E$. It can be seen that the examples provided in Section 4 are all special cases of the representation in Proposition 5. Notice however that, as $I$ does not satisfy Monotonicity, the weight $\rho_{x, y}(A)$ is not necessarily in $[0,1]$ (although it is necessarily in $(0,1)$ for the event $E$ ).

We conclude the dicussion of this case by showing that, when Axiom CB is added to the axioms in Proposition 5, biseparability holds for all the events in $\mathscr{E}$.

Corollary 2. $\succcurlyeq$ satisfies the assumptions of Proposition 5 and CB if and only if there exists a nonempty set $\mathscr{E} \subseteq \Sigma$ such that the function $\rho: X_{u \uparrow}^{2} \times \Sigma \rightarrow \mathbb{R}$ obtained in Proposition 5 satisfies: $F \in \mathscr{E}$ iff $\rho_{x, y}(F)=\rho_{F} \in(0,1)$ for all $(x, y) \in X_{u \uparrow}^{2} \cdot{ }^{2}$

Proof of Corollary 2. If $\succcurlyeq$ satisfies CB, then for all $F \in \mathscr{E}, \succcurlyeq$ is $F$-biseparable and by Lemma 1, all the representations $V_{F}$ are cardinally equivalent: $V_{E} \approx V_{F} \equiv V$ for all $E, F \in \mathscr{E}$. Therefore, if we consider $x F y$ for $F \in \mathscr{E}$, then by Prop. $4, V(x F y)=\rho_{F} u(x)+\left(1-\rho_{F}\right) u(y)$, for some $\rho_{F} \in(0,1)$. So, for any $F \in \mathscr{E}$ and for any $(x, y) \in X_{u \uparrow}^{2}$, let $\rho_{x, y}(F)=\rho_{F}$. Next for any $A \in \Sigma \backslash \mathscr{E}$ and $(x, y) \in X_{u \uparrow}^{2}$, let $\rho_{x, y}(A) \equiv \frac{I(u \circ(x A y))-u(y)}{u(x)-u(y)}$ when $u(x)>u(y)$ and $\rho_{x, y}(A) \equiv \frac{1}{2}$ when $u(x)=u(y)$. Therefore, Eq. (11) holds for all $A \in \Sigma$.

\footnotetext{
${ }^{2}$ The uniqueness properties are those stated in footnote 1 , plus uniqueness of $\mathscr{E}$.
} 
To see the necessity of CB, consider $E, F \in \mathscr{E}$. Eq. (11), the properties of $\rho_{x, y}(\cdot)$ and Prop. 2 imply that $\succcurlyeq$ is $E$ - and $F$-biseparable, and by the uniqueness properties of the biseparable representation we have $u_{F} \approx u \approx u_{E}$. But then $V_{E}$ and $V_{F}$ are cardinally equivalent, so by Lemma $1, E$ and $F$ are average-equivalent and hence CB holds.

\section{D.1.3 Monotone preferences}

In this section, we impose the (global) Monotonicity axiom and we derive a fully subjective version of the Monotone, Bernoullian and Archimedean (MBA) preferences of CGMMS.

Axiom (Monotonicity - M). For all $f, g \in \mathscr{F}, f(s) \succcurlyeq g(s)$ for all $s \in S$ implies $f \succcurlyeq g$.

This axiom immediately implies monotonicity of the representation $V$ (i.e. $f(s) \succcurlyeq g(s)$ for all $s \in S$ implies $V(f) \geq V(g)$ ), and hence also the fact that $\rho_{x, y}(\cdot)$ is (non-negative and) a capacity on $\Sigma$. It is straightforward to adapt to proof of Proposition 5 to obtain the following stronger result:

Proposition 6. $\succcurlyeq$ satisfies Axioms $P, C, M$, and there exists an $O N$ event $E \in \Sigma$ if and only if there is a representation $V$ of $\succcurlyeq$ that can be written as $V=I \circ u$, where $u: X \rightarrow \mathbb{R}$ is a $\tau$-continuous, nonconstant function, and $I: B_{0}(\Sigma, u(X)) \rightarrow \mathbb{R}$ is a normalized, continuous and monotone functional such that for all binary acts $x$ Ay with $x \succcurlyeq y$ and $A \in \Sigma$,

$$
I(u \circ(x A y))=\rho_{x, y}(A) u(x)+\left(1-\rho_{x, y}(A)\right) u(y)
$$

for some $\rho: X_{u \uparrow}^{2} \times \Sigma \rightarrow \mathbb{R}$ such that: 1) $\rho_{x, y}(\cdot)$ is a capacity on $\Sigma$ for all $(x, y) \in X_{u \uparrow}^{2}$;2) $\rho_{x, y}(E)=\rho_{E} \in$ $(0,1)$ for all $(x, y) \in X_{u \uparrow}^{2}$ and for some $E \in \Sigma^{3}$

Proposition 6 also shows that an MBA representation can be obtained without imposing the Risk Independence axiom with respect to an objective mixture structure. ${ }^{4}$ On the other hand, adding a standard independence property (see Axiom SI in Supp. App. D.4) allows us to prove that the functional $I$ obtained in Proposition 6 is linear. By standard arguments, we can then prove that $I$ is an expectation. We thus obtain an alternative axiomatization of subjective expected utility.

As observed in Remark 1, CGMMS show that events $A$ which are unambiguous according to their definition are such that $p(A)=q(A)=\rho_{x, y}(A)=\hat{\rho}$ for all $p, q$ in the set $C$ which rep-

\footnotetext{
${ }^{3}$ The uniqueness properties are identical to those stated in footnote 1.

${ }^{4}$ That is, if $x, y, z \in X$ and $\lambda \in[0,1]$, then (with + denoting the objective mixture operation) $x \succcurlyeq y \Longleftrightarrow \lambda x+(1-\lambda) z \succcurlyeq$ $\lambda y+(1-\lambda) z$.
} 
resents perceived ambiguity. It is then clear that if $A$ is unambiguous and nontrivial, then $\succcurlyeq$ is $A$-biseparable (with $\rho(A)=\hat{\rho}$ ), so that it follows by Proposition 2 that $A$ satisfies ON.

\section{D.1.4 Stake-independent willingness-to-bet}

We now ask when, as in the biseparable preferences of Ghirardato and Marinacci (2001), the capacity $\rho_{x, y}$ is independent of the payoffs. Since we dropped Certainty Independence, we do not have constant linearity of the functional $I$. From a behavioral viewpoint, constant linearity means that the DM's valuation of an act is independent of the scale and position of its outcomes. Without this property, the DM's willingness to bet on an event depends on the magnitude of the prizes, which is often considered a behavioral bias (Bénabou, 2015), but arises naturally in our world. Adapting the arguments in Section 4.1 of CGMMS, outcome independence is restored by assuming a version of the Certainty Independence axiom that only applies to binary acts.

Let $\mathscr{F}_{b}$ be the the set of all binary acts. We require Certainty Independence only on $\mathscr{F}_{b}$ :

Axiom (Binary Certainty Independence - BCI). For all $f, g \in \mathscr{F}_{b}, x \in X$, and $\gamma \in(0,1)$,

$$
f>g \Longleftrightarrow \gamma f \oplus(1-\gamma) x>\gamma g \oplus(1-\gamma) x
$$

A simple adaptation of the argument in Ghirardato and Marinacci (2001, Thm. 9) then yields:

Proposition 7. $\succcurlyeq$ satisfies the assumptions of Proposition 6 and BCI if and only if in the representation obtained in Proposition 6 we have $\rho_{x, y}(A)=I\left(1_{A}\right)$ for all $(x, y) \in X_{u \uparrow}^{2}$ and for all $A \in \Sigma$.

\section{D.1.5 Ambiguity Hedging and Uncertainty Averse Preferences}

One particularly interesting class of monotone preferences is the class of "Uncertainty Averse" preferences (UAP) axiomatized by Cerreia-Vioglio, Maccheroni, Marinacci, and Montrucchio (2011) in the AA setting. It generalizes most of the pre-existing models, such as MEU, Variational and Confidence preferences. The only restriction of the DM's ambiguity attitude is given by the UA axiom of Schmeidler (1989), which, as discussed in Remark 2, in our context is naturally replaced by the AH axiom. As discussed in Ghirardato and Marinacci (2002), Ambiguity Hedging is a property which embodies a strong form of aversion to ambiguity. DMs may display ambiguity aversion while violating it. (Recent experimental evidence on this is provided by Kuzmics, Rogers, and 
Zhang (2019), who find subjects that strictly prefer act $f$ (or $g$ ) to an act which in their setting reproduces the 1/2:1/2 subjective mixture of $f$ and $g$.)

We start by observing that, in the presence of the axioms of Proposition 2, Axiom AH is equivalent to quasi-concavity of $V$.

Proposition 8. Let $\succcurlyeq$ be a binary relation that has an E-biseparable representation $V$ for some $E \in \Sigma$. Then, $\succcurlyeq$ satisfies Axiom $A H$ if and only if the representation $V$ is also quasi-concave with respect to $\oplus$; that is, for any $f, g, h \in \mathscr{F}$ such that $V(f) \geq V(h)$ and $V(g) \geq V(h)$, and for all $\lambda \in[0,1]$, $V(\lambda f \oplus(1-\lambda) g) \geq V(h)$.

Proof of Proposition 8. We start by showing that our axioms imply the following Archimedean Continuity property: for any $f, g, h \in \mathscr{F}$, the sets $U=\{\alpha \in[0,1]: \alpha f \oplus(1-\alpha) g \succcurlyeq h\}$ and $L=\{\alpha \in$ $[0,1]: h \succcurlyeq \alpha f \oplus(1-\alpha) g\}$ are closed. Since all acts in $\mathscr{F}$ are simple acts, given $f, h, g \in \mathscr{F}$ there is a finite partition $\Pi$ that makes all three acts measurable. Moreover, the mixture $\lambda f \oplus(1-\lambda) g$ is measurable w.r.t. to $\Pi$, for all $\lambda \in[0,1]$. Now, consider a sequence $\left\{\alpha_{n}\right\} \in U$ such that $\alpha_{n} \rightarrow \alpha$. Then $\left(\alpha_{n} f \oplus\left(1-\alpha_{n}\right) g\right) \succcurlyeq h$ for all $n$, and $\left(\alpha_{n} f \oplus\left(1-\alpha_{n}\right) g\right) \rightarrow(\alpha f \oplus(1-\alpha) g)$. So by Axiom C, $(\alpha f \oplus(1-\alpha) g) \succcurlyeq h$, implying $\alpha \in U$.

Given this property, the quasi-concavity of the functional $V$ obtained in Proposition 2 now follows from a simple adaptation of the argument in the proof of Lemma 60 in Cerreia-Vioglio et al. (2011). The opposite direction is standard.

When we further add the Monotonicity axiom, so that $V=I \circ u$ as shown in Proposition 6, Axiom AH is then equivalent to quasi-concavity of $I$.

Proposition 9. Let $\succcurlyeq$ be a binary relation that has a representation $(I, u)$ as in Proposition 6. Then, $\succcurlyeq$ satisfies Axiom AH if and only if the functional I is also quasiconcave. ${ }^{5}$

Proof of Proposition 9. Given the representation of Proposition 6, Axiom AH is equivalent to $I(u \circ$ $f)=I(u \circ g)$ implies $I\left(u \circ\left(\frac{1}{2} f \oplus \frac{1}{2} g\right)\right) \geq I(u \circ g)$. By the properties of $\oplus$, we have $I\left(\frac{1}{2} u \circ f+\frac{1}{2} u \circ g\right) \geq$ $I(u \circ g)$, so that by induction

$$
I\left(\frac{k}{2^{n}} u \circ f+\frac{2^{n}-k}{2^{n}} u \circ g\right) \geq I(u \circ g)
$$

\footnotetext{
${ }^{5}$ The uniqueness properties are identical to those stated in footnote 1.
} 
$n=0,1,2, \ldots$ and $0 \leq k \leq 2^{n}$. The set $\left\{\frac{k}{2^{n}}, n=0,1,2, \ldots, 0 \leq k \leq 2^{n}\right\}$ is dense in [0,1], so for all $\lambda \in$ $(0,1)$ it contains a sequence converging to $\gamma$, namely $\lim _{j \rightarrow \infty} \frac{k_{j}}{2^{n_{j}}}=\gamma$. By continuity of $I$, for all $\lambda \in(0,1)$,

$$
I(\lambda u \circ f+(1-\lambda) u \circ g)=\lim _{j \rightarrow \infty} I\left(\frac{k_{j}}{2^{n_{j}}} u \circ f+\left(1-\frac{k_{j}}{2^{n_{j}}}\right) u \circ g\right) \geq I(u \circ g)
$$

Then $I$ is quasiconcave. The opposite direction is standard.

It then follows from the results of Cerreia-Vioglio et al. (2011), that the functional $I$ obtained in the proposition can be written as follows:

$$
I(u \circ f)=\inf _{p \in \Delta(S)} G\left(\int u \circ f d p, p\right)
$$

where $G: u(X) \times \Delta(S) \rightarrow(-\infty, \infty]$ is linearly continuous. ${ }^{6}$ So Proposition 9 provides a fully subjective axiomatization of UAP.

\section{D.2 Trivial events and the cardinality of the representation}

In Section 3.2, we argue that the existence of an event which satisfies the ON property is necessary for Definition 1 to correctly identify utility midpoints. Here we prove more generally that the existence of nontrivial uncertainty is necessary to obtain the cardinality of utility via a biseparability property like Eq. (3) (formalizing an insight of Ellsberg (1954); see also Remark 8 in Ghirardato and Marinacci (2001)). For, when all events are either null or universal a representation satisfying Eq. (3) is not cardinal, even if the preference satisfies Axioms P and C.

Fact 2. Suppose that $\succcurlyeq$ satisfies Axioms $P$ and $C$. If all events in $\Sigma$ are either null or universal, then any functional $V: \mathscr{F} \rightarrow \mathbb{R}$ representing $\succcurlyeq$ is such that for all $x>y$ and all $A \in \Sigma, V(x A y)=$ $\rho(A) V(x)+(1-\rho(A)) V(y)$, with $\rho(A)=0$ if $A$ is null and $\rho(A)=1$ if $A$ is universal.

Proof of Fact 2. Given $\mathrm{P}$ and C, Lemma 3 implies that for each $f \in \mathscr{F}$ there exists $x \in X$ with $x \sim f$ and denote it $c_{f}$. By standard arguments, there exists a continuous $u: X \rightarrow \mathbb{R}$ representing $\succcurlyeq$ on $X$, unique up to a monotonic nondecreasing transformation. Define $V: \mathscr{F} \rightarrow \mathbb{R}$ as $V(f) \equiv$ $u\left(c_{f}\right)$. Then, $V$ represents $\succcurlyeq$. Consider $A \in \Sigma$ and $x>y$, then, either $x A y \sim x$ or $x A y \sim y$. In the former case, $V(x A y)=V(x)$, so, letting $\rho(A)=1$, we have $V(x A y)=\rho(A) V(x)+(1-\rho(A)) V(y)$. A

\footnotetext{
${ }^{6}$ A function $G: T \times \Delta(S) \rightarrow(-\infty, \infty]$ with $T \subseteq \mathbb{R}$ is said to be linearly continuous if the map $a \mapsto \inf _{p \in \Delta(S)} G\left(\int a d p, p\right)$ from $B_{0}(\Sigma, T)$ is extended-valued and continuous.
} 
similar argument holds for the latter case with $\rho(A)=0$. Clearly, $V$ is unique up to a monotonic nondecreasing transformation and $\rho(A) \in\{0,1\}$ for all $A \in \Sigma$.

That is, any representation $V$ of $\succcurlyeq$ satisfies Eq. (3) (with $\rho \in\{0,1\}$ ). But then so do its monotonic increasing transformations.

\section{D.3 Calculations for example 1}

Let $S=\left\{s_{1}, s_{2}\right\}, X=[0,10], u(x)=x$ hence $u(X)=[0,10]$, and define

$$
V(x E y)=1_{u(x) \leq 5}[0.3 x+0.7 y]+1_{x>5}\left[\rho_{x} u(x)+\left(1-\rho_{x}\right) y\right]
$$

with $\rho_{x}=0.2+0.02(10-x)$. We first show that the preference represented by $V$ satisfies betwise monotonicity. There are three cases to check:

- $(5 \geq x, y, z)$ : Then $V(x E z)=0.3 x+0.7 z$, hence $x \succcurlyeq y$ if and only if $x E z \succcurlyeq y E z$ for all $x, y \succcurlyeq z$. If $z \succcurlyeq x, y, V(z E x)=0.3 z+0.7 x$ and $x \succcurlyeq y$ if and only if $z E x \succcurlyeq z E y$.

- $(x>5 \geq y): V(x E z)=\rho_{x} x+\left(1-\rho_{x}\right) z$ and $V(y E z)=0.3 y+0.7 z$ for all $x, y \succcurlyeq z$. For $x=10$, $0.2 \cdot 10+0.8 z \geq 0.3 y+0.7 z$, for all $z \leq y \leq 5$. For $x=5,0.3 \cdot 5+0.7 z \geq 0.3 y+0.7 z$ for all $z \leq y \leq 5$.

- $(x, y>5): V(x E z)=\rho_{x} x+\left(1-\rho_{x}\right) z$ and $V(y E z)=\rho_{y} y+\left(1-\rho_{y}\right) z$ for all $x, y \succcurlyeq z$. Let $x \succcurlyeq y$ and we need to prove $V(x E z)-V(y E z)=\rho_{x} x-\rho_{y} y+z\left(\rho_{y}-\rho_{x}\right) \geq 0$, By definition $\rho_{y} \geq \rho_{x}$, so it is sufficient to prove $\rho_{x} x-\rho_{y} y \geq 0$, but this can be verified applying the definition of $\rho_{x}$ and $\rho_{y}$.

The preference $\succcurlyeq$ violates ordered bisymmetry. For instance, take $x>5$ and $z, z^{\prime}<5$ such that $c_{x E z}, c_{x E z^{\prime}}>5$. Then, for $z>z^{\prime}$ :

$$
\begin{aligned}
V\left(c_{x E z} E c_{z^{\prime} E y}\right) & =\rho_{c_{x E z}}\left(\rho_{x} x+\left(1-\rho_{x}\right) z\right)+\left(1-\rho_{c_{x E z}}\right)\left(0.3 z^{\prime}+0.7 y\right) \\
& \neq \rho_{c_{x E z^{\prime}}}\left(\rho_{x} x+\left(1-\rho_{x}\right) z^{\prime}\right)+\left(1-\rho_{c_{x E z^{\prime}}}\right)(0.3 z+0.7 y) \\
& =V\left(c_{x E z^{\prime}} E c_{z E y}\right)
\end{aligned}
$$


With $x=7, z=6, z^{\prime}=5, y=3, V(x E z)=0.26 \cdot 7+0.74 \cdot 6=6.26, V\left(z^{\prime} E y\right)=0.3 \cdot 5+0.7 \cdot 3=3.6$, $V\left(x E z^{\prime}\right)=0.26 \cdot 7+0.74 \cdot 5=5.52, V(z E y)=0.28 \cdot 6+0.72 \cdot 3=3.84$. Thus,

$$
\begin{aligned}
V\left(c_{x E z} E c_{z^{\prime} E y}\right) & =\rho_{6.26} V(x E z)+\left(1-\rho_{6.26}\right) V\left(z^{\prime} E y\right) \\
& =0.2748 \cdot 6.26+0.7252 \cdot 3.6 \\
& =4.3302 \\
& \neq 4.3265 \\
& =0.2895 \cdot 5.52+0.7105 \cdot 3.84 \\
& =\rho_{5.52} V\left(x E z^{\prime}\right)+\left(1-\rho_{5.52}\right) V(z E y) \\
& =V\left(c_{x E z^{\prime}} E c_{z E y}\right)
\end{aligned}
$$

a violation of bisymmetry.

\section{D.4 MTI and Independence}

Here, we show that Proposition 3 implies that Axiom MTI is (in the presence of the other axioms) equivalent to the adaptation to subjective mixtures of the standard independence axiom:

Axiom (Subjective mixture Independence - SI). For all $f, g, h \in \mathscr{F}$ and all $\lambda \in[0,1]$,

$$
f \succcurlyeq g \Longrightarrow \lambda f \oplus(1-\lambda) h \succcurlyeq \lambda g \oplus(1-\lambda) h
$$

Corollary 3. Given a locally biseparable preference, it satisfies Axiom MTI if and only if it also satisfies Axiom SI.

Proof of Corollary 3. If MTI holds, $u\left(c_{\lambda f \oplus(1-\lambda) g}\right)=u\left(\lambda c_{f} \oplus(1-\lambda) c_{g}\right)=\lambda u\left(c_{f}\right)+(1-\lambda) u\left(c_{g}\right)$. So, by the definition of $V, V(\lambda f \oplus(1-\lambda) g)=\lambda V(f)+(1-\lambda) V(g)$. Suppose now that $f \succcurlyeq g$. Then, $u\left(c_{f}\right) \geq$ $u\left(c_{g}\right)$ and therefore $\lambda u\left(c_{f}\right)+(1-\lambda) u\left(c_{h}\right) \geq \lambda u\left(c_{g}\right)+(1-\lambda) u\left(c_{h}\right)$ for any $\lambda \in[0,1]$ and any $h \in \mathscr{F}$. By definition of subjective mixture, $u\left(\lambda c_{f} \oplus(1-\lambda) c_{h}\right) \geq u\left(\lambda c_{g} \oplus(1-\lambda) c_{h}\right)$; by MTI, $u\left(c_{\lambda f \oplus(1-\lambda) h}\right)=$ $u\left(\lambda c_{f} \oplus(1-\lambda) c_{h}\right) \geq u\left(\lambda c_{g} \oplus(1-\lambda) c_{h}\right)=u\left(c_{\lambda g \oplus(1-\lambda) h}\right)$, and therefore $\lambda f \oplus(1-\lambda) h \succcurlyeq \lambda g \oplus(1-\lambda) h$. If SI holds, then $\mathrm{P}$ and the definitions of $c_{f}, c_{g}$ imply $\lambda f \oplus(1-\lambda) g \sim \lambda c_{f} \oplus(1-\lambda) g \sim \lambda c_{f} \oplus(1-\lambda) c_{g}$.

Axiom MTI is weaker than the original AA's Reversal of Order. Indeed, MTI does not imply 
"reduction of compound lotteries" (ROCL) even when outcomes are objective lotteries, $X=\Delta(Z)$. In the original AA's setting with an objective mixture operator + , ROCL is $\alpha \delta_{p}+(1-\alpha) \delta_{q} \sim \alpha p+(1-$ $\alpha) q$. AA’s Reversal of Order implies ROCL. However, with MTI, $\alpha \delta_{p}+(1-\alpha) \delta_{q} \equiv \alpha p \oplus_{A}(1-\alpha) q \sim$ $\alpha p \oplus(1-\alpha) q \not \alpha p+(1-\alpha) q$.

That is, $\succcurlyeq$ represented by $V$ can satisfy MTI but $V(f) \neq \sum_{s \in S} \sum_{z \in Z} w(z) f(s)(z) p(s)$, where $f(s)(z)$ is the probability assigned to $z$ by the lottery $f(s)$.

\section{D.5 Additional examples for Subsection 5.2}

Another stark example of a preference which satisfies Eq. (8) globally is the following. Let $X=\mathbb{R}_{++}$, $u(x)=\ln x$, and $V(f)=\mathbb{E}_{p}[\ln (f)]$, for some $p \in \Delta(S)$. It is natural to interpret $V$ as an expected utility preference with a logarithmic Bernoulli index, hence not sensitive to ambiguity. However, as it satisfies Axiom UA, it would traditionally be interpreted as reflecting aversion to ambiguity.

Finally, we have an example of a locally biseparable preference which satisfies Eq. (9). Suppose $\succcurlyeq$ is a locally biseparable preference represented by a $V$ with $u(x)=e^{x}$, but that for some event $A \in \Sigma$, $\succcurlyeq$ is not $A$-biseparable. Specifically, suppose that for any $x \succcurlyeq y$,

$$
V(x A y)=\rho_{\{x, y\}}(A) u(x)+\left(1-\rho_{\{x, y\}}(A)\right) u(y)
$$

with $\rho_{\{x, y\}}(A)=0.5$ whenever $x-y>2$ and $\rho_{\{x, y\}}(A)=0.64537$ when $x-y \leq 2$. Consider now $x=4, y=1$ and $z=3.35544$. Then, given $f=x A y$ and $g=z$, we have $f \sim g$, since $V(x A y)=$ $0.5 e^{4}+0.5 e^{1}=e^{3.35544}=u(z)$. On the other hand, we have that $V\left(\frac{1}{2} f+\frac{1}{2} g\right)=0.64537 \cdot e^{\left(\frac{1}{2} 4+\frac{1}{2} 3.35544\right)}+$ $0.35463 \cdot e^{\left(\frac{1}{2} 1+\frac{1}{2} 3.35544\right)}=e^{3.35544}=u(z)=V(f)$, while $V\left(\frac{1}{2} f \oplus \frac{1}{2} g\right)=0.64537 \cdot\left(\frac{1}{2} e^{4}+\frac{1}{2} e^{3.35544}\right)+$ $0.35463 \cdot\left(\frac{1}{2} e^{1}+\frac{1}{2} e^{3.35544}\right)=e^{3.47905}>e^{3.3554}$. So we have

$$
\frac{1}{2} f+\frac{1}{2} g \sim g \quad \text { and } \quad \frac{1}{2} f \oplus \frac{1}{2} g>g
$$

\section{D.6 A general framework for mixtures of menu-acts}

We now briefly show that our approach to subjective mixtures can also be used in settings where acts map states of the world to menus of consequences. Such settings can be used to model, among others, preference for flexibility Dekel, Lipman, and Rustichini (2001, henceforth DLR), 
dynamic (in)consistency due to temptation and other behavioral problems (Gul and Pesendorfer, 2001), or costly learning about the decision framework (e.g. Ergin and Sarver, 2010). Our approach allows us to provide a more general theory, as it does not restrict subjective states to be vNM expected utilities, even though we impose some limits on the DM's preference for flexibility. An advantage of this approach is the possibility of replacing the Independence axiom, which is common in models of menu-choice (e.g. DLR, Gul and Pesendorfer, 2001; Dekel, Lipman, and Rustichini, 2009), with a more transparent assumption concerning subjective mixtures.

Working in the setting described in Section 2, we follow Nehring (1999) and Ghirardato (2001) and let acts be maps $f: S \rightarrow \mathscr{K}$, where $\mathscr{K}$ is a suitably chosen family of non-empty subsets of $X$ (called menus). We impose first of all Axiom P, thus asking the DM to be able to rank all acts in $\mathscr{F}$. Call crisp any act $f$ such that $f(s)$ is a singleton for all $s \in S$; i.e., a traditional Savage act. Denote by $\mathscr{F}_{c}$ the set of all crisp acts. The next axiom is our key assumption on preferences:

Axiom (Crisp Equivalence - CE). For all $A \in \mathscr{K}$, there exists $x \in X$ such that $x \sim A$.

Using intuitive notation, we denote by $c_{A}$ the crisp equivalent of menu $A$. It should be observed that we are not making specific assumptions on the relation between $c_{A}$ and the elements of $A$ : $c_{A}$ could belong to $A$, as it is the case for instance in the Desire for Commitment axiom of Dekel et al. (2009) or in the Contingencywise Dominance axiom of Ghirardato (2001), but that is not necessary. Yet, as mentioned above, Axiom CE limits the scope of the DM's preference for flexibility by ruling out preference orderings which rank some menu above all singletons. ${ }^{7}$

If we add Axiom $\mathrm{D}$ to Axioms $\mathrm{P}$ and $\mathrm{CE}$, we obtain that for any $f \in \mathscr{F}$, there is a crisp act $f_{c} \in \mathscr{F}_{c}$ such that $f_{c} \sim f$. Because of this result, if we now impose Axioms C and CB (restricted to the set $\mathscr{F}_{c}$ ), we can follow the steps in the previous sections to endow $\mathscr{F}$ with a subjective mixture structure defined by $\frac{1}{2} f_{c} \oplus \frac{1}{2} g_{c}$, for every $f, g \in \mathscr{F}$. This structure allows us to recast in our framework existing axiomatizations, providing a fully subjective representations of preferences over menu-acts.

One issue that arises in this context is how to define subjective mixtures of menus. On one hand, given two menus $A, B \in \mathscr{K}$ and our notion of subjective mixture $\oplus$, we can follow DLR in defining the mixture $\bar{\oplus}$ of $A$ and $B$ as follows: $\lambda A \bar{\oplus}(1-\lambda) B \equiv\{\lambda x \oplus(1-\lambda) y: x \in A, y \in B\}$; i.e., a menu consisting of the $z \in X$ that are subjective mixtures of elements of $A$ and $B$. On the other

\footnotetext{
${ }^{7}$ This does not rule out preference for flexibility altogether. For instance, Kreps (1979)'s model of preference for flexibility is compatible with Axiom CE.
} 
hand, treating $A$ and $B$ as constant acts, we can also employ a mixture operation in the spirit of Section 5.1, $\lambda c_{A} \oplus(1-\lambda) c_{B}$. In general, these two notions of (subjective) menu mixture will be different. However, there is a natural restriction on preferences which makes them equivalent: Axiom MTI for constant menu-acts.

Axiom (Menu Mixture Timing Indifference - MMTI). For all $A, B \in \mathscr{K}$ and all $\lambda \in[0,1]$,

$$
\lambda c_{A} \oplus(1-\lambda) c_{B} \sim \lambda A \bar{\oplus}(1-\lambda) B
$$

The discussion of axiom MTI in the previous section applies mutatis mutandis: the evaluations of $A$ and $B$ (i.e., determining their crisp equivalent) are performed separately, and they are also performed separately from the act of evaluating their mixture.

To provide a more structured interpretation, we recall that preferences over menus are typically used to model situations where the DM may not perfectly know — say, because of "unforeseen contingencies"- her future tastes (see, e.g., DLR). That is, the DM faces two types of uncertainty: the objective states (described by $s \in S$ ) as well as "subjective states" that correspond to her final preference orderings. There are therefore two notions of "timing" that one can envision: timing with respect to the resolution of the objective uncertainty, and timing with respect to the resolution of the "subjective-state" uncertainty. Axiom MTI in Subsection 5.1 stipulates that the DM be indifferent between (subjective) mixtures that are resolved before the observation of $s$ and mixtures that are resolved after the observation of $s .{ }^{8}$ Symmetrically, Axiom MMTI requires that the DM be indifferent between mixtures that resolve before (left-hand side) and after (right-hand side) the "realization" of the DM's "subjective state." The indifference in MMTI can also be interpreted as "neutrality to contingent planning," in the spirit of Ergin and Sarver (2010). Indeed, the left-hand side of the indifference does not entail the anticipation of (state-contingent) secondperiod choice, since it is the mixture of two singletons. In contrast, the evaluation of the mixture of two menus in the right-hand side forces the individual to consider second-period choice contingent on each subjective state.

Reasoning along the same lines as Corollary 3, it is simple to show that imposing Axiom MMTI is equivalent to requiring that the DM satisfies DLR's Independence Axiom with respect to (either notion of) menu mixtures. The advantage of MMTI lies in its more direct interpretation. DLR

\footnotetext{
${ }^{8}$ Once again, our reference to timing in discussing subjective mixtures is purely suggestive.
} 
observe that in their setting with a given objective mixture structure, the Independence Axiom forces the DM to be indifferent between objective ex-ante and ex-post randomization, thus restricting their "subjective states" to expected utility preferences. In contrast, in our setting one could obtain a fully subjective DLR-style representation. In such representation, the "subjective states" would be preferences whose representing utilities are not necessarily affine (i.e. non-EU) with respect to an objective mixture structure, but are affine with respect to the subjective mixture structure $\oplus$. Thus, the adoption of subjective mixtures allows for a wider scope of representations, even in the presence of strong separability conditions like Axiom MMTI.

Our approach also allows weakening the DLR independence axiom, hence non-indifference towards the timing of randomization. Analogously to Subsection 5.1, relaxations of MMTI which capture preference for early or late randomization are directly mapped to global properties (i.e. concavity and convexity) of the representation. For example, following Ergin and Sarver (2010), consider the function:

$$
u(A)=\max _{G \in \mathscr{G}} \int_{\Omega} \max _{x \in A} \mathbb{E}_{\mu}(U \mid G)(\omega)(x) \mu(d \omega)-c(G)
$$

where $A \in \mathscr{K}$ and $\omega \in \Omega$ is a "subjective state" à la DLR, which entails the preference representation $U(\omega)(\cdot)$ on $X$. Each $G \in \mathscr{G}$ represents a contemplation strategy (i.e., a $\sigma$-algebra of the subjective state space $\Omega$ ) and, given the subjective probability $\mu \in \Delta(\Omega), \mathbb{E}_{\mu}(U \mid G)(\omega)(x)$ is the conditional expectation of $U(\omega)(x)$ with respect to $\mu$ and the $\sigma$-algebra $G$. In a setting with objective randomization +, Ergin and Sarver (2010) show that a preference over menus represented by Eq. (15) with convex $u$ is characterized by a form of translation invariance and the following property, dubbed "Aversion to Contingent Planning": for all $A, B \in \mathscr{K}, A \sim B$ implies $A \succcurlyeq \lambda A+(1-\lambda) B$.

In our subjective mixture setting, the convexity of $u$ can be guaranteed by a single axiom relaxing MMTI which can be analogously interpreted as aversion to contingent planning: a preference for early mixture $\lambda c_{A} \oplus(1-\lambda) c_{B} \succcurlyeq \lambda A \bar{\oplus}(1-\lambda) B$. Moreover, as for the extension of DLR discussed above, in our setting subjective states are not necessarily EU preferences. 


\section{References}

Roland Bénabou. The economics of motivated beliefs. Revue d'économie politique, 125(5):665685, 2015.

Simone Cerreia-Vioglio, Fabio Maccheroni, Massimo Marinacci, and Luigi Montrucchio. Uncertainty averse preferences. Journal of Economic Theory, 146(4):1275-1330, 2011.

Eddie Dekel, Barton L Lipman, and Aldo Rustichini. Representing preferences with a unique subjective state space. Econometrica, 69(4):891-934, 2001.

Eddie Dekel, Barton L Lipman, and Aldo Rustichini. Temptation-driven preferences. The Review of Economic Studies, 76(3):937-971, 2009.

Daniel Ellsberg. Classic and current notions of "measurable utility". The Economic Journal, 64 (255):528-556, 1954.

Haluk Ergin and Todd Sarver. A unique costly contemplation representation. Econometrica, 78(4): 1285-1339, 2010.

Paolo Ghirardato. Coping with ignorance: unforeseen contingencies and non-additive uncertainty. Economic Theory, 17(2):247-276, 2001.

Paolo Ghirardato and Massimo Marinacci. Risk, ambiguity, and the separation of utility and beliefs. Mathematics of Operations Research, 26(4):864-890, 2001.

Paolo Ghirardato and Massimo Marinacci. Ambiguity made precise: A comparative foundation. Journal of Economic Theory, 102(2):251-289, 2002.

Simon Grant and Ben Polak. Mean-dispersion preferences and constant absolute uncertainty aversion. Journal of Economic Theory, 148(4):1361 - 1398, 2013.

Faruk Gul and Wolfgang Pesendorfer. Temptation and self-control. Econometrica, 69(6):14031435, 2001.

David M Kreps. A representation theorem for "preference for flexibility". Econometrica, 47(3): 565-577, 1979.

Christoph Kuzmics, Brian Rogers, and Xiannong Zhang. Is Ellsberg behavior evidence of ambiguity aversion? SSRN Paper 3437331, 2019. 
Klaus Nehring. Preference for flexibility in a Savage framework. Econometrica, 67(1):101-119, 1999.

David Schmeidler. Subjective probability and expected utility without additivity. Econometrica, 57(3):571-587, 1989. 